\title{
Novel fragile $X$ syndrome 2D and 3D brain models based on human isogenic FMRP-KO iPSCs
}

\author{
Carlo Brighi ${ }^{1,2}$, Federico Salaris (10 ${ }^{1,3}$, Alessandro Soloperto ${ }^{1}$, Federica Cordella ${ }^{1,2}$, Silvia Ghirga ${ }^{1,4}$, Valeria de Turris (1) \\ Maria Rosito ${ }^{1}$, Pier Francesca Porceddu ${ }^{5}$, Chiara D'Antoni ${ }^{1,2}$, Angelo Reggiani ${ }^{5}$, Alessandro Rosa $\mathbb{C}^{1,3}$ and \\ Silvia Di Angelantonio (10 ${ }^{1,2}$
}

\begin{abstract}
Fragile X syndrome (FXS) is a neurodevelopmental disorder, characterized by intellectual disability and sensory deficits, caused by epigenetic silencing of the FMR1 gene and subsequent loss of its protein product, fragile $X$ mental retardation protein (FMRP). Delays in synaptic and neuronal development in the cortex have been reported in FXS mouse models; however, the main goal of translating lab research into pharmacological treatments in clinical trials has been so far largely unsuccessful, leaving FXS a still incurable disease. Here, we generated 2D and 3D in vitro human FXS model systems based on isogenic FMR1 knock-out mutant and wild-type human induced pluripotent stem cell (hiPSC) lines. Phenotypical and functional characterization of cortical neurons derived from FMRP-deficient hiPSCS display altered gene expression and impaired differentiation when compared with the healthy counterpart. FXS cortical cultures show an increased number of GFAP positive cells, likely astrocytes, increased spontaneous network activity, and depolarizing GABAergic transmission. Cortical brain organoid models show an increased number of glial cells, and bigger organoid size. Our findings demonstrate that FMRP is required to correctly support neuronal and glial cell proliferation, and to set the correct excitation/inhibition ratio in human brain development.
\end{abstract}

\section{Introduction}

Fragile X syndrome (FXS), first described by J. Purdon Martin and Julia Bell in 1943 as a mental defect following an X-linked inheritance pattern ${ }^{1}$, represents the second cause of inherited intellectual disability after the Down syndrome, and the most prevalent one in males ${ }^{2,3}$. FXS patients are characterized by cognitive impairment, defective communication, abnormal sensory reactivity, anxiety, hyperactivity, gaze aversion, and impulsivity ${ }^{4}$. The etiology of FXS lies in an abnormal trinucleotide repeat

\footnotetext{
Correspondence: Alessandro Rosa (alessandro.rosa@uniroma1.it) or

Silvia Di Angelantonio (silvia.diangelantonio@uniroma1.it)

${ }^{1}$ Center for Life Nano- \& Neuro-Science, Istituto Italiano di Tecnologia, Viale Regina Elena 291, 00161 Rome, Italy

${ }^{2}$ Department of Physiology and Pharmacology, Sapienza University of Rome, P.le A. Moro 5, 00185 Rome, Italy

Full list of author information is available at the end of the article

These authors contributed equally: Carlo Brighi, Federico Salaris, Alessandro Soloperto

These authors contributed equally:Alessandro Rosa, Silvia Di Angelantonio Edited by A. Verkhratsky
}

(CGG) expansion in the $5^{\prime} \mathrm{UTR}$ of the fragile X mental retardation 1 (FMR1) gene, which leads to its hypermethylation, transcriptional silencing, and subsequent lack of the fragile $\mathrm{X}$ mental retardation protein (FMRP) ${ }^{5}$.

As a widely expressed RNA binding protein in the brain, FMRP plays a pivotal role in the stability and translational regulation of hundreds of mRNAs involved in synaptic protein synthesis ${ }^{6}$, synaptic plasticity, and neuronal development ${ }^{7-9}$. Absence or incorrect expression of FMRP is directly linked with alterations in dendritic spine architecture, synaptogenesis, and neural connectivity, as suggested by profound alterations of anatomical development in the cortex of Fmr1 knock-out (Fmr1 KO) mice $^{5,10}$. In vivo studies on Fmr1 KO mice have revealed many aspects of FXS pathophysiology, providing fundamental information on the functionality of FMRP and its involvement in neurogenesis, neuronal maturation, and synaptic plasticity formation ${ }^{11-13}$. However, despite the Fmr1 KO mouse represents a fundamental resource in

\section{(c) The Author(s) 2021}

\footnotetext{
(c) Open Access This article is licensed under a Creative Commons Attribution 4.0 International License, which permits use, sharing, adaptation, distribution and reproduction cc) in any medium or format, as long as you give appropriate credit to the original author(s) and the source, provide a link to the Creative Commons license, and indicate if changes were made. The images or other third party material in this article are included in the article's Creative Commons license, unless indicated otherwise in a credit line to the material. If material is not included in the article's Creative Commons license and your intended use is not permitted by statutory regulation or exceeds the permitted use, you will need to obtain permission directly from the copyright holder. To view a copy of this license, visit http://creativecommons.org/licenses/by/4.0/.
} 
understanding the molecular pathways altered in FXS, physiological and evolutionary species-specific differences have hampered translating these results from rodents to humans, raising the need for a humanized FXS model. In the last decade, human induced Pluripotent Stem Cells (hiPSCs) have emerged as useful tools for modeling neurodevelopmental disorders, including FXS $^{14-17}$. Moreover, introducing specific disease-causing genomic alterations into the hiPSC line of choice by gene editing allows minimizing possible confounding effects due to the different genetic background of patient-derived hiPSC lines ${ }^{18}$. Notably, genome editing allows generating isogenic pools of wild-type and mutant hiPSC clones, providing a straightforward approach for unmasking phenotypic and functional alterations characteristic of complex diseases, such as Alzheimer's disease ${ }^{19}$, Parkinson's disease ${ }^{20}$, Huntington's disease ${ }^{21}$, and $\mathrm{FXS}^{21}$.

Three-dimensional (3D) brain models provide significant advantages compared to conventional bidimensional (2D) cell cultures in terms of more properly recapitulating: tissue complexity ${ }^{22}$, gene expression and protein synthesis ${ }^{23,24}$, differentiation and migration behaviors ${ }^{25}$, cellular morphology and cell-to-cell and cellextracellular matrix contacts ${ }^{26-28}$. To this regard, despite hiPSC-derived 3D brain organoids have been developed for modeling many neurodevelopmental diseases ${ }^{29}$, including autism ${ }^{30,31}$, to the best of our knowledge their use to model FXS has not been yet pursued.

To investigate the function of FMRP in cortical brain development, we genetically engineered a healthy control hiPSC line by truncating the FMR1 gene through CRISPR/Cas9 gene editing, thus generating an isogenic FMRP-KO hiPSC line. We report the phenotypical and functional characterization of FMRP-deficient hiPSCderived cortical neurons, which display altered gene expression and impaired differentiation when compared with the isogenic control. Moreover, we generated and characterized 3D self-assembled brain organoids. Interestingly, since the brain organoid model recapitulates the early stages of human cortical development, all the phenotypical perturbations caused by FMRP deficiency, in terms of neuro-glia development, can be evaluated in a critical developmental period otherwise hardly accessible for investigations in humans. Moreover, the presence of intrinsically heterogeneous cell populations within brain organoids allowed assessing simultaneously the impact of FMRP loss on neuronal network and glial cells.

\section{Results}

\section{Generation and characterization of FMRP KO hiPSCs}

In order to produce an in vitro FXS model system made of isogenic mutant and control hiPSC lines, we generated a FMRP knock-out line by gene editing in a FMRP wildtype genetic background. The human FMR1 genomic locus is depicted in Fig. 1A. We designed a strategy, based on the CRISPR/Cas9 system, to block the production of FMRP at the level of transcription. Taking into consideration the presence of an antisense non-coding RNA, the production of multiple transcripts from the FMR1 gene and the apparent absence of downstream alternative transcription start sites (Supplementary Fig. S1), the strategy to produce a knock-out mutant was based on the insertion of a selection gene spliced in frame with the first FMR1 exon. We designed two guide RNAs (gRNA\#1 and gRNA\#2) directed against this region and a donor construct with homology arms. Upon homology-directed repair, a selection cassette encoding for a self-cleavage peptide (T2A), a puromycin resistance gene, and a cleavage and polyadenylation signal was inserted in frame after the first three codons of exon 2. With this design, as a result of transcription and splicing, a transcript encoding for the puromycin resistance protein (puromycin-Nacetyltransferase) would be produced, while no FMR1 mRNA could be generated.

The fact that the FMR1 gene is on the X-chromosome facilitated the knock-out strategy in male cells (the hiPSC line $\mathrm{WTI}^{32}$ ), as only one allele needed to be edited. After transfection and selection with puromycin, PCR analysis on genomic DNA with specific primers showed effective integration of the cassette in hiPSCs co-transfected with the Cas9, the donor construct and gRNA\#2 (Fig. 1A). We next isolated single clones from the gRNA\#2-transfected population. As shown in Fig. 1B, PCR analysis on genomic DNA confirmed the specific integration of the cassette in several clones. We then performed on these clones a RTPCR analysis with primer pairs flanking splicing junctions (Fig. 1C). All clones showed the amplification product of the primer set specific for the spliced knock-out construct (first row in Fig. 1C). Conversely, the unedited spliced mRNA could be detected in the parental line only, as demonstrated by the analysis with reverse primers annealing beyond exon 1 (Fig. 1C, second to fourth row). Consistently, in the same cells no FMRP protein was detectable by Western blot or immunostaining (Fig. 1D). Complete absence of FMR1 transcript and protein was then confirmed in cortical neurons generated by gene edited hiPSC differentiation at day 10 and 25 (Figs. 1E, $\mathrm{F}$ and $2 \mathrm{~A}$ ).

One gene edited clone (clone \#5; hereafter FMRP-KO) and parental isogenic hiPSCs (hereafter FMRP-WT) were differentiated to cortical neurons using a conventional protocol and 2D culture conditions. Acquisition of a neural cortical fate was induced by dual SMAD inhibition followed by the block of Hedgehog signaling with cyclopamine (Supplementary Fig. S2A) ${ }^{33,34}$. Exit from pluripotency and acquisition of neuronal character was assessed by qRT-PCR analysis of pluripotency (OCT4), neural progenitor cells (PAX6), and neuronal (TUJ1) 


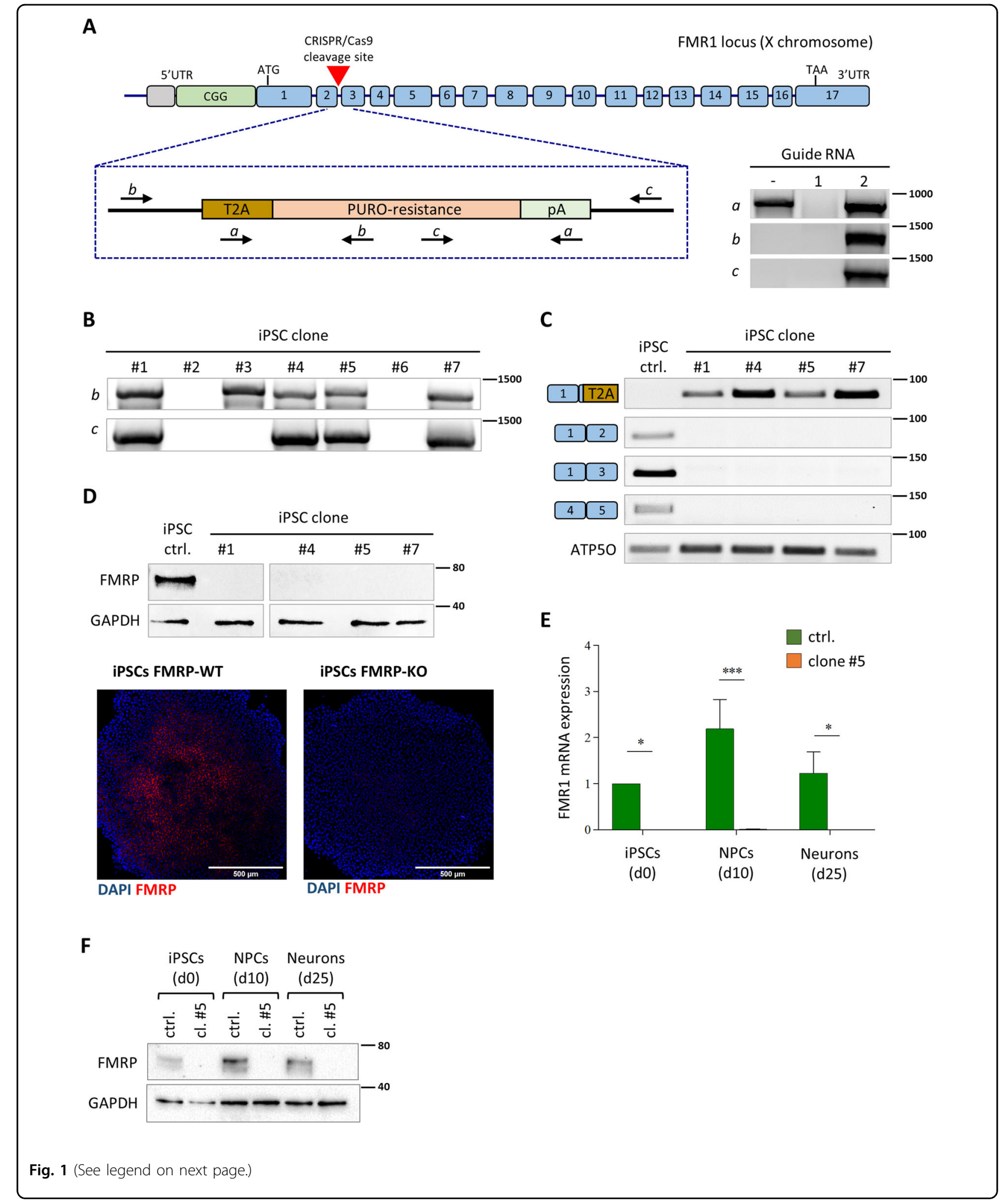

FMR1 locus (X chromosome)

markers during the first 25 days of differentiation (Fig. 2A, left). Subsequent analysis up to 70 days of differentiation showed expression of neuronal markers (TUJ1, PAX6,
TBR1, TBR2; Fig. 2A, middle) and late upregulation of astrocytic markers (GFAP, S100ß; Fig. 2A, right) in both genotypes (FMRP-WT top; FMRP-KO, bottom). 
(see figure on previous page)

Fig. 1 Generation and validation of FMRP knock-out hiPSCs. A Top: schematic representation of the human FMR1 locus and strategy for gene editing. The red triangle represents the site of cleavage by Cas9. The selection cassette, shown below, encodes for the self-cleavage peptide (T2A) and the puromycin resistance gene (PURO-resistance) and contains a cleavage and polyadenylation signal (pA). Primers used for the PCR analysis on genomic DNA are depicted as arrows. Bottom: PCR analysis on genomic DNA of hiPSCs transfected with the donor construct only (first lane) or cotransfected with the Cas9 and gRNA\#1 or gRNA\#2 (second and third lanes). B PCR analysis on genomic DNA of the indicated hiPSC clones with primers indicated in panel A. C RT-PCR analysis on RNA isolated from the indicated hiPSC clones with primers spanning exon-exon junctions. The housekeeping gene ATP5O was used as an internal control. D Top: Western blot analysis of FMRP expression in the indicated hiPSC clones. GAPDH was used as loading control. Bottom: immunostaining analysis in parental and FMRP-KO hiPSCs. Red: FMRP; blue: DAPI. Scale bar: $500 \mu$ m. E Real-time qRT-PCR analysis of cortical neurons derived from parental and FMRP-KO hiPSCs ( $n=3$ differentiation batches; Student's $t$ test; paired; two tails; ${ }^{*} p<$ $\left.0.05,{ }^{* *} p<0.001\right)$. F Western blot analysis of FMRP expression in cortical neurons derived from parental and FMRP-KO hiPSCs. GAPDH was used as loading control.

Immunostaining analysis confirmed expression of PAX6 at the neural rosette stage and lack of FMRP in the FMRP$\mathrm{KO}$ line (Fig. 2B). We then analyzed a panel of early and late neural markers over multiple differentiation experiments to assess whether the absence of FMRP could lead to defects in differentiation at early stages. At day 10 of differentiation, corresponding to the late neural induction phase and initial acquisition of the neural progenitor cell character, we noticed a slight increase of $S O X 2$ expression in FMRP-KO cells, with a substantial degree of variability among different biological replicates (Fig. 2C). However, no significant change was observed for other early neural markers (FOXG1, PAX6, SOX9, and NESTIN). At day 25, corresponding to a stage in which differentiating cells that are acquiring a neuronal character exit from the cell cycle, no significant difference was detected in PAX6 and SOX2 expression between FMRP-WT and FMRP-KO differentiating cultures (Fig. 2D), suggesting that the slight increase of SOX2 observed at day 10 was a transient effect. At this stage, however, we noticed a significant increase in the levels of the astrocyte marker GFAP and concomitant decrease of the neuronal precursor marker TBR2 in FMRP-KO cells, which also showed a slight but not significant increase of another astrocyte marker, $S 100 \beta$ (Fig. 2D). Taken together, these results validate a novel FMRP knock-out hiPSC line, generated by gene editing, showing early imbalance of neuronal and astrocyte markers during cortical differentiation.

\section{FMRP-KO cortical neurons display increased excitatory transmission during in vitro maturation}

As cortical hyperexcitability is one of the hallmarks of FXS, and neuronal hyperexcitability has been reported both in slices and culture of $F m r 1 \mathrm{KO}$ mouse model ${ }^{8}$, we characterized, through confocal analysis of immunofluorescence signals and functional recordings of calcium dynamics, the development of the glutamatergic and the GABAergic systems in 2D cortical cultures differentiated from FMRP-WT and FMRP-KO hiPSCs.

At a late stage of development and maturation a mixed population of differentiating cells was achieved, with the simultaneous presence of neural progenitors, neurons, and glial cells. In both FMRP-WT and KO cultures at day 54 , consistent with previous findings ${ }^{33}$, immunofluorescence analysis of neuronal and glial markers indicated that GFAP staining was barely detectable (not shown), while the large majority of cells were positive for the neuronal marker MAP2 (Fig. 3A, B). The presence of glutamatergic and GABAergic pre-synaptic specialization was demonstrated in both genotypes by the positive staining of neurons for both presynaptic VGLUT1 (Fig. 3A) and GAD67 (Fig. 3B), respectively. Moreover, positive staining for PSD95 (Fig. 3A) demonstrated the presence of post-synaptic glutamatergic specialization. When comparing FMRP-WT and FMRP-KO cultures at day 54, while we did not find any difference in presynaptic GAD67 staining (Fig. 3B), we observed that FMRP-KO iPSC derived neuronal cultures display higher number of pre- (VGLUT1) and post- (PSD95) synaptic glutamatergic components (puncta; Fig. 3C, left,), with increased colocalization compared to FMRP-WT (Fig. 3C, right), indicating faster excitatory synaptic development (Fig. 3A, see also puncta details at higer magnification in Supplementary Fig. S3), thus indicating that during cortical neuron maturation FMRP-KO neurons display an accelerated development of glutamatergic system (Fig. 3C).

At day 70, we observed the development of astrocytes in the $2 \mathrm{D}$ cultues as revealed by the positive staining of both FMRP-WT and FMRP-KO cultures for GFAP (Fig. 4A) and $\mathrm{S} 100 \beta$ (Supplementary Fig. S4A). Side-by-side comparison indicated another early FXS phenotype in FMRP$\mathrm{KO}$, consisting of an accelerated specification of GFAPpositive astrocytes as revealed by GFAP staining (Fig. 4A, B). Conversely, we observed that, as for presynaptic GABAergic specialization (GAD67), the expression levels of pre- (VGLUT1) and post- (PSD95) synaptic glutamatergic markers became similar (Fig. 4C, D), suggesting that the increase in glutamatergic network was transient in FMRP-KO cultures (Fig. 4E, see Supplementary Fig. S4B for higher magnification).

Strikingly, at day 70, we observed a functional FXS phenotype when measuring spontaneous intracellular 
A

FMRP-WT (2D)
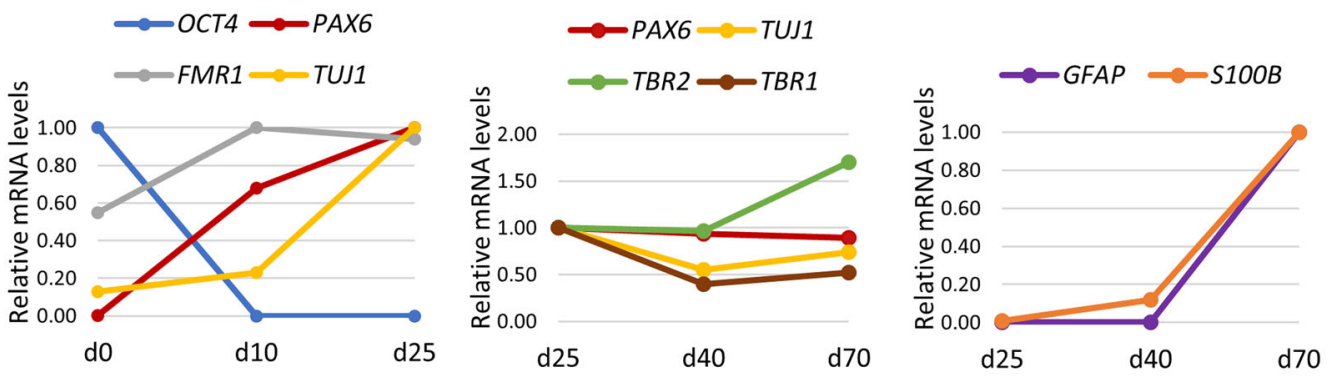

FMRP-KO (2D)
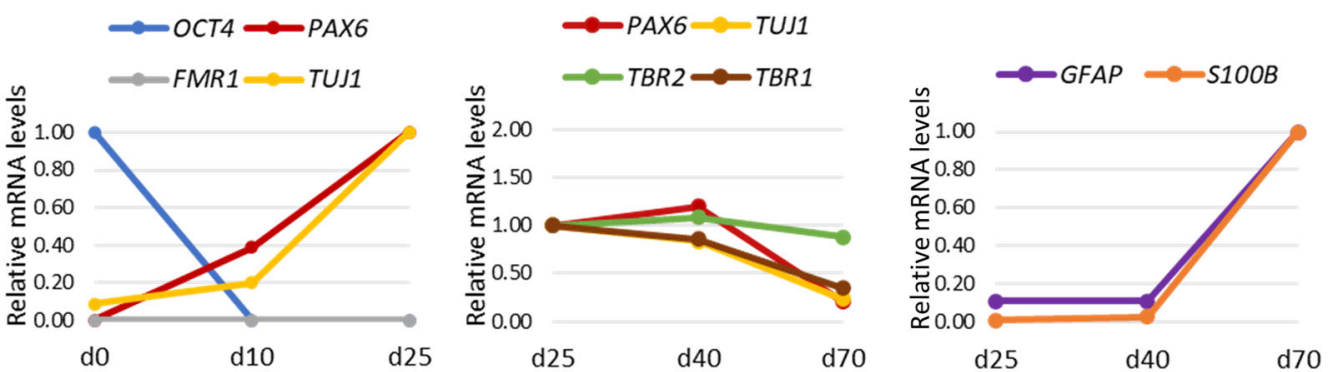

B FMRP WT (2D) day 25

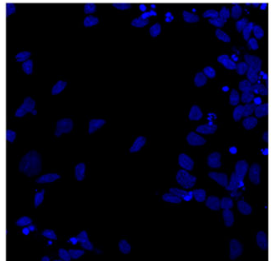

DAPI

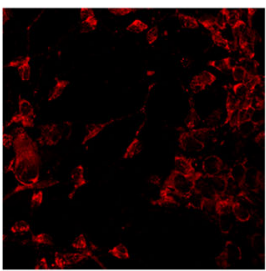

FMRP

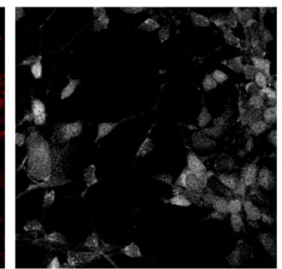

PAX6

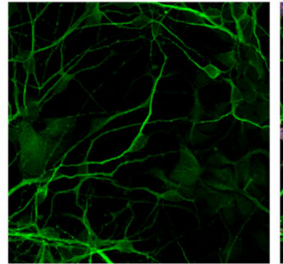

MAP2

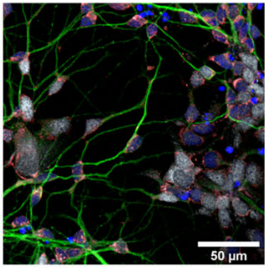

Merge

FMRP KO (2D) day 25

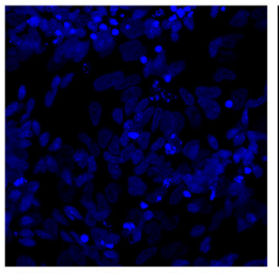

DAPI

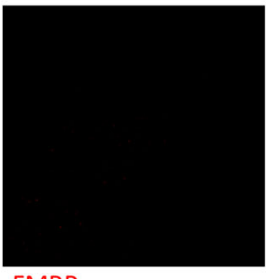

FMRP

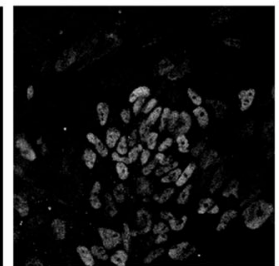

PAX6

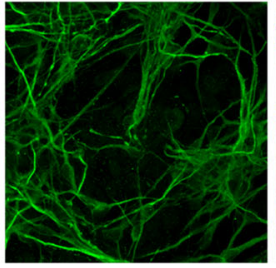

MAP2

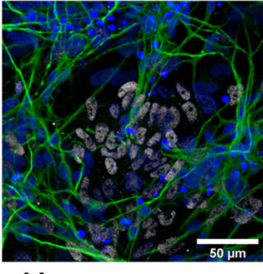

Merge
C

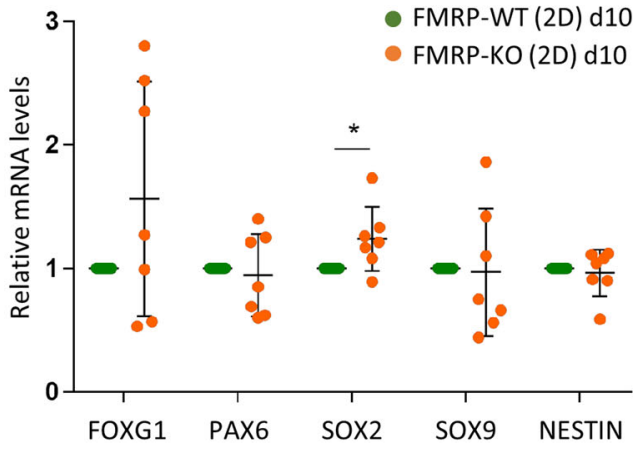

D

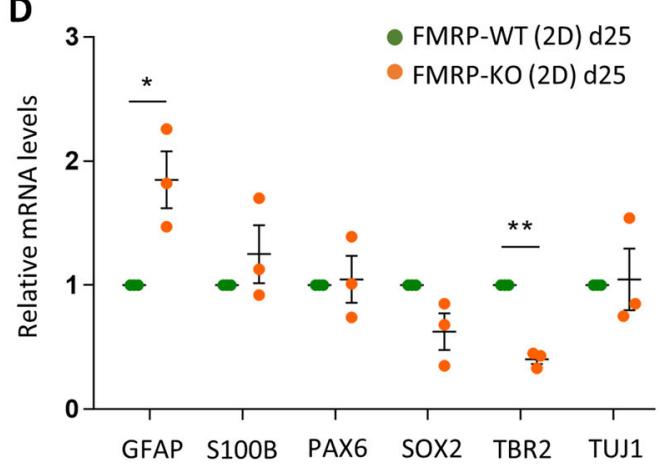

Fig. 2 Differentiation of FMRP knock-out and isogenic control hiPSCs into cortical neurons. A Real-time gRT-PCR analysis of the indicated markers expression in differentiating FMRP-WT (top) and FMRP-KO (bottom) cells at the indicated time points. B Representative images of immunostaining for FMRP (red), PAX6 (white), and MAP2 (green) expression in FMRP-WT and FMRP-KO cells at day 25. Scale bar: $50 \mu \mathrm{m}$. C, D Real-time qRT-PCR analysis of the indicated markers expression in FMRP-WT and FMRP-KO cells at day 10 (C) and 25 (D) of differentiation (FMRP-KO vs FMRP-WT; Student's $t$ test; paired; ${ }^{*} p<0.05,{ }^{* *} p<0.01 ; n=3$ differentiation batches). 


\section{A $\quad$ FMRP-WT (2D) d54}
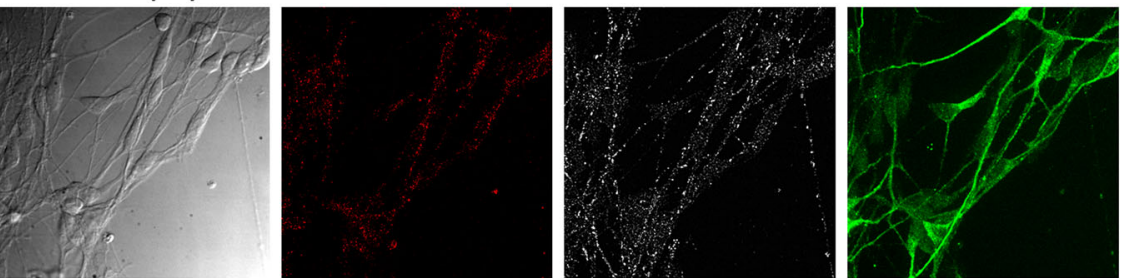

VGLUT1

PSD95

MAP2

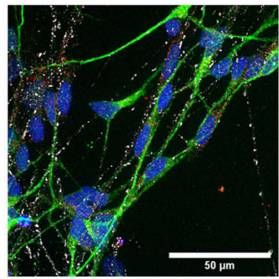

Merge

FMRP-KO (2D) d54
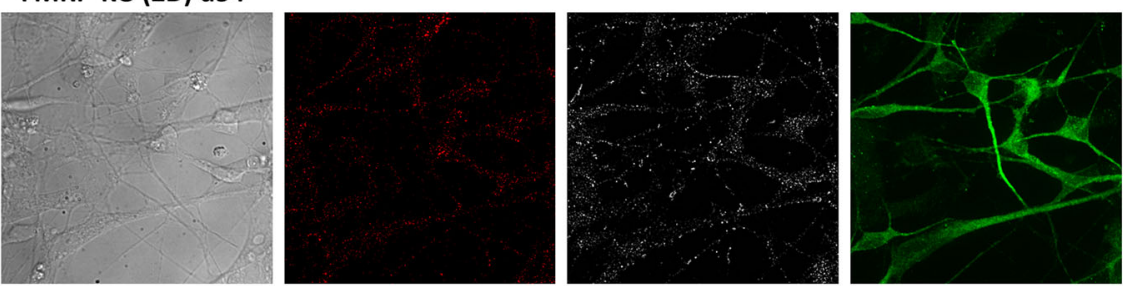

VGLUT1

PSD95

MAP2

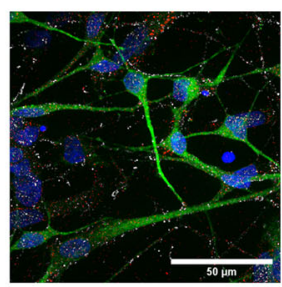

Merge

B

\section{FMRP-WT (2D) d54}
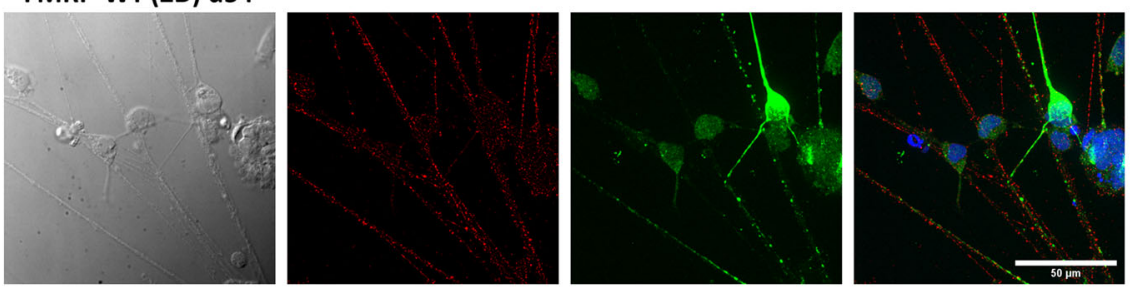

GAD67

MAP2

Merge

FMRP-KO (2D) d54
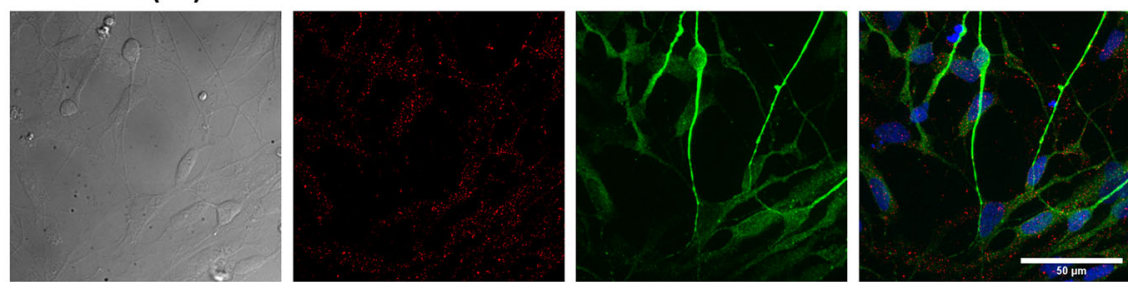

GAD67

MAP2

Merge

C
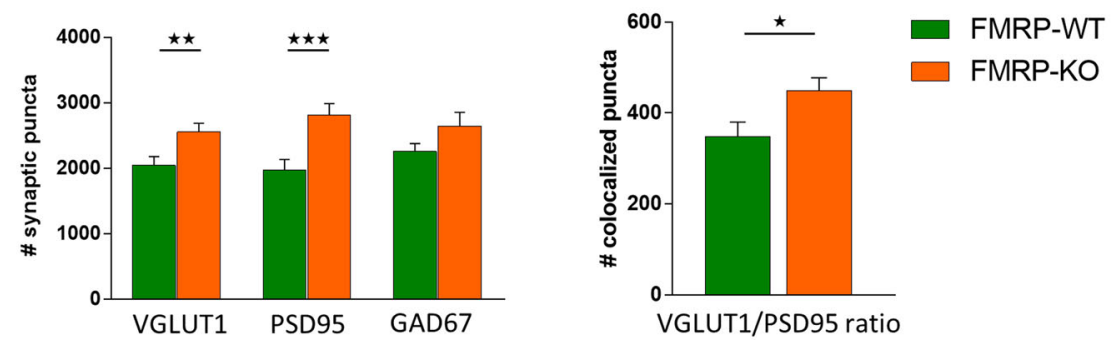

Fig. 3 Immunofluorescence analysis of 2D cortical cultures at day 54. A Representative immunofluorescence and transmitted images of glutamatergic synapses at day 54 in 2D cortical cultures differentiated from FMRP-WT (top) and FMRP-KO (bottom) hiPSCs. Glutamatergic synaptic puncta are identified as positive for VGLUT1 (pre-synaptic, red) and PSD95 (post-synaptic, white), respectively. Nuclei were stained with DAPI (blue) and dendrites with MAP2 (green). Scale bar: $50 \mu \mathrm{m}$. B Representative immunofluorescence and transmitted images of GABAergic synapses at day 54 in $2 \mathrm{D}$ cortical cultures differentiated from FMRP-WT (top) and FMRP-KO (bottom) hiPSCs. GABAergic pre-synaptic puncta are shown as positive for GAD67 (red). C Bar charts representing (left) the quantification of glutamatergic and GABAergic synaptic puncta (VGLUT1 puncta WT 2050 \pm 130, KO $2550 \pm 140,{ }^{* *} p<0.01$; PSD95 puncta WT $1980 \pm 160, \mathrm{KO} 2810 \pm 180,{ }^{* * *} p<0.001 \mathrm{MW}$ test; GAD67 puncta WT 2250 $\pm 130, \mathrm{KO} 2640 \pm 210, p=0.11$; $n=30 / 2 \mathrm{FOV}$ s/differentiation batches for each genotype) and (right) VGLUT1/PSD95 colocalization ratio in both genotypes (VGLUT1/PSD95 colocalized puncta WT $350 \pm 30, \mathrm{KO} 450 \pm 30,{ }^{*} p<0.05 \mathrm{MW}$ test; $n=30 / 2 \mathrm{FOVs} /$; differentiation batches for each genotype). 


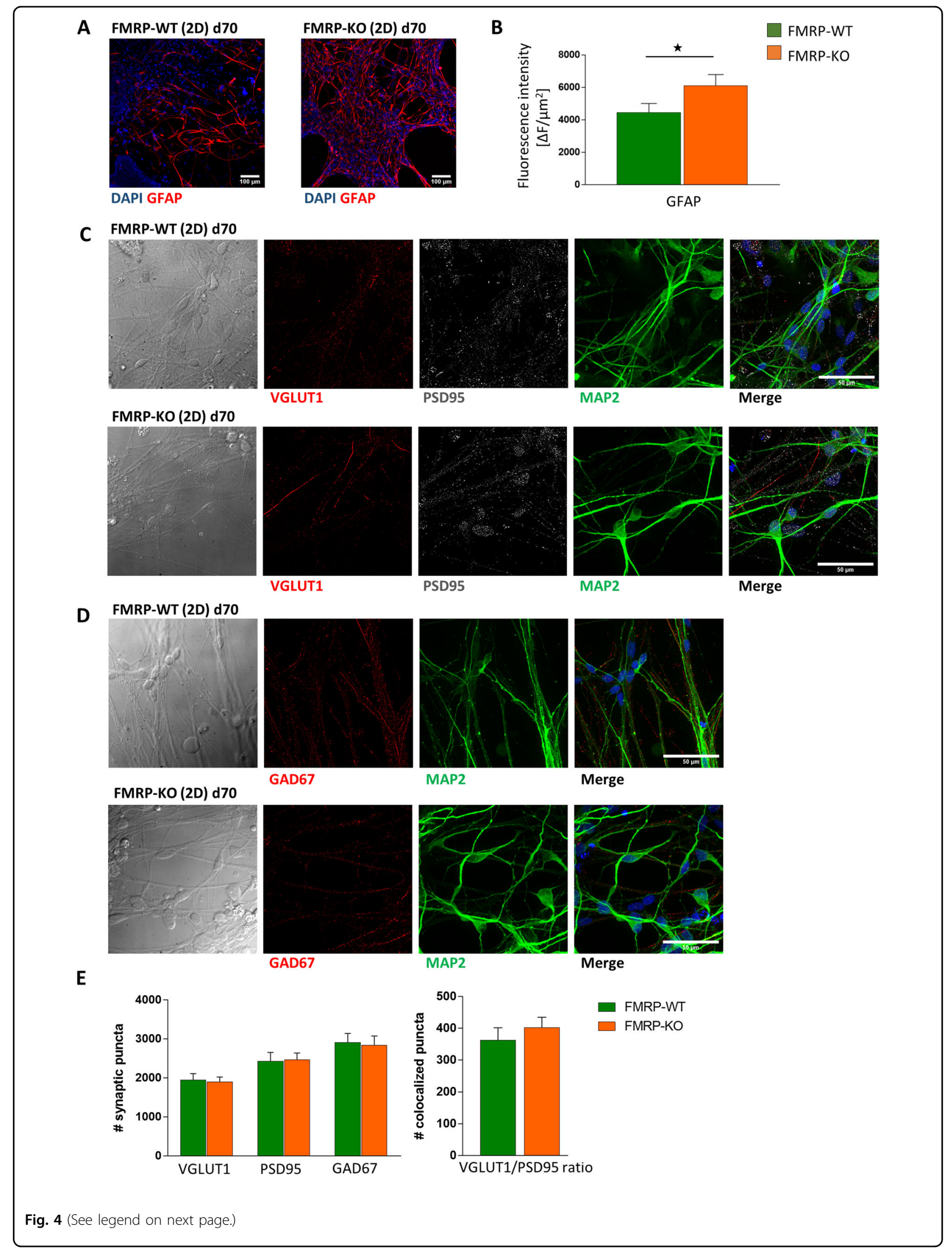


(see figure on previous page)

Fig. 4 Immunofluorescence analysis of 2D cortical cultures at day 70. A Representative images of differentiated 2D cortical cultures from FMRPWT and FMRP-KO hiPSCs at day 70 immunolabeled with anti-GFAP antibody (red) and stained with DAPI for nuclei visualization (blue). Scale bar: $100 \mu \mathrm{m}$. B Quantification of mean GFAP fluorescence signal intensity ( ${ }^{*} p<0.05 \mathrm{KO}$ vs WT, MW test; $n=20 / 2 / 1$, FOVs/ cultures/differentiation batches). C Representative immunofluorescence and transmitted images of glutamatergic synapses at day 70 in $2 \mathrm{D}$ cortical cultures differentiated from FMRP-WT (top) and FMRP-KO (bottom) hiPSCs. Glutamatergic synaptic puncta are identified as positive for VGLUT1 (pre-synaptic, red) and PSD95 (post-synaptic, white), respectively. Nuclei were stained with DAPI (blue) and dendrites with anti-MAP2 antibody (green). Scale bar: $50 \mu m$. D Representative immunofluorescence and transmitted images of GABAergic synapses at day 70 in 2D cortical cultures differentiated from FMRP-WT (top) and FMRP-KO (bottom) hiPSCs. GABAergic pre-synaptic puncta are shown as positive for GAD67 (red). E Bar charts representing (left) the quantification of glutamatergic and GABAergic synaptic puncta (VGLUT1 puncta WT 1950 \pm 160, KO $1900 \pm 130, p=0.87$, MW test; PSD95 puncta WT $2430 \pm 220, \mathrm{KO} 2470 \pm 170, p=0.9 ;$ GAD67 puncta WT $2910 \pm 230 \mathrm{KO} 2840 \pm 230, p=0.83$, MW test; $n=30 / 2 \mathrm{FOVs} /$ differentiation batches for each genotype) and (right) VGLUT1/PSD95 colocalization, in both genotypes (VGLUT1/PSD95 WT $360 \pm 40, \mathrm{KO} 400 \pm 30, p=0.43 ; n=30 / 2$ FOVs/ differentiation batches for each genotype).

calcium dynamics at network level and the glutamate and GABA evoked calcium responses at the level of single neuron. We recorded spontaneous calcium transients in the 2D neuronal networks loaded with Fluo-4M, using a customized digital fluorescence microscope with a $4 \mathrm{~Hz}$ sampling of a large field of view $(260 \times 200$ micron $)$, that allowed to monitor a large number of cells at the same time. Data from a 5-minute time-lapse recordings were collected and analyzed through a custom-made algorithm (Supplementary Fig. S5) designed to recognize cells, select active cells, sort calcium events, dividing cells in two groups characterized by the occurrence of calcium events with fast or slow rise time, respectively (Fig. 5A, B), and finally extract functional properties such as amplitude, frequency, kinetic parameters, and network synchronicity $^{34,35}$.

For each field of view (see Supplementary videos 1 and 2), using 2 seconds value as rising time threshold, we divided active cells in FAST (Fig. 5A top) and SLOW (Fig. 5A bottom) population. As represented in the dot plots, the proportion of both FAST and SLOW active cells were similar in FMRP-WT and FMRP-KO cultures (Fig. 5C, D). As reported in Fig. 5E the spontaneous activity of FAST cells, most probably neurons, was significantly stronger in FXS cultures; indeed both the amplitude (Fig. $5 \mathrm{E}$ left) and the frequency (Fig. 5E middle and right) of calcium events were significantly higher in FMRP-KO neurons respect to FMRP-WT neurons, with no difference in synchronized firing, thus suggesting the possible overexcitability of the FXS cortical network. On the other hand, analyzing calcium traces extracted from the SLOW population, likely ascribable to glial cells and differentiating neuronal cells, we found that FMRP-KO cells displayed spontaneous calcium events characterized by higher amplitude (Fig. 5F left) but similar frequency (Fig. 5F middle and right) and synchronization with respect to FMRP-WT cells, suggesting that also the astrocytic network is more active in FXS cortical cultures.

As the cortical network activity and excitability are strictly dependent on the balance of excitatory and inhibitory transmission, we evaluated the potency and the sign of glutamatergic and gabaergic transmission monitoring in 2D cultures calcium transients evoked by a local and brief agonist application through a puffer pipette (Fig. $5 \mathrm{G})$. Glutamate application $(2 \mathrm{mM}, 100 \mathrm{~ms}, 10$ p.s.i.) evoked intracellular calcium responses, indicating functional expression of glutamate receptors (Fig. $5 \mathrm{H}$, insert). As depicted in Fig. 5H glutamatergic responses were significantly higher in FMRP-KO cells with respect to FMRP-WT, suggesting that even though the number of pre- and post- synaptic markers was similar in the two genotypes, the neuronal depolarization mediated by glutamatergic receptor activation is strongly upregulated in our FXS model, probably contributing to the increased network activity. Neuronal hyperexcitability and seizure susceptibility are common in FXS patients and animal models, and they are believed to result from an imbalance between excitatory and inhibitory drives in intracortical circuits, with reduced inhibition as key mechanism for circuit hyperexcitability. As defective GABAergic inhibition may arise from a depolarizing GABA effect, and depolarizing GABA is typical of immature neurons, we tested if GABA application may induce a transient calcium increase in our cultures at day 70. As reported in Fig. 5I calcium transients evoked by puffer application of GABA ( $1 \mathrm{mM} ; 100 \mathrm{~ms} 10 \mathrm{psi}$ ) were significantly higher in FMRP-KO neurons with respect to FMRP-WT; moreover, while GABA application induced a depolarizing effect on $30 \pm 3 \%$ of FMRP-WT neurons $(n=33 / 6$ cells/FOV; Fig. 5 I, insert), in FMRP-KO cultures $75 \pm 3 \%$ ( $n=57 / 6$ cells/ FOV; Fig. 5I, insert) of neurons responded to GABA with a depolarization-mediated calcium transient, indicating that FMRP-KO cultures displayed an immature phenotype.

Altogether these data suggest that FMRP-KO cultures are characterized by a FXS-related functional phenotype with pronounced hyperexcitability mediated by increased glutamatergic transmission and decreased GABAergic inhibition, possibly due to immature depolarizing GABAergic response. 


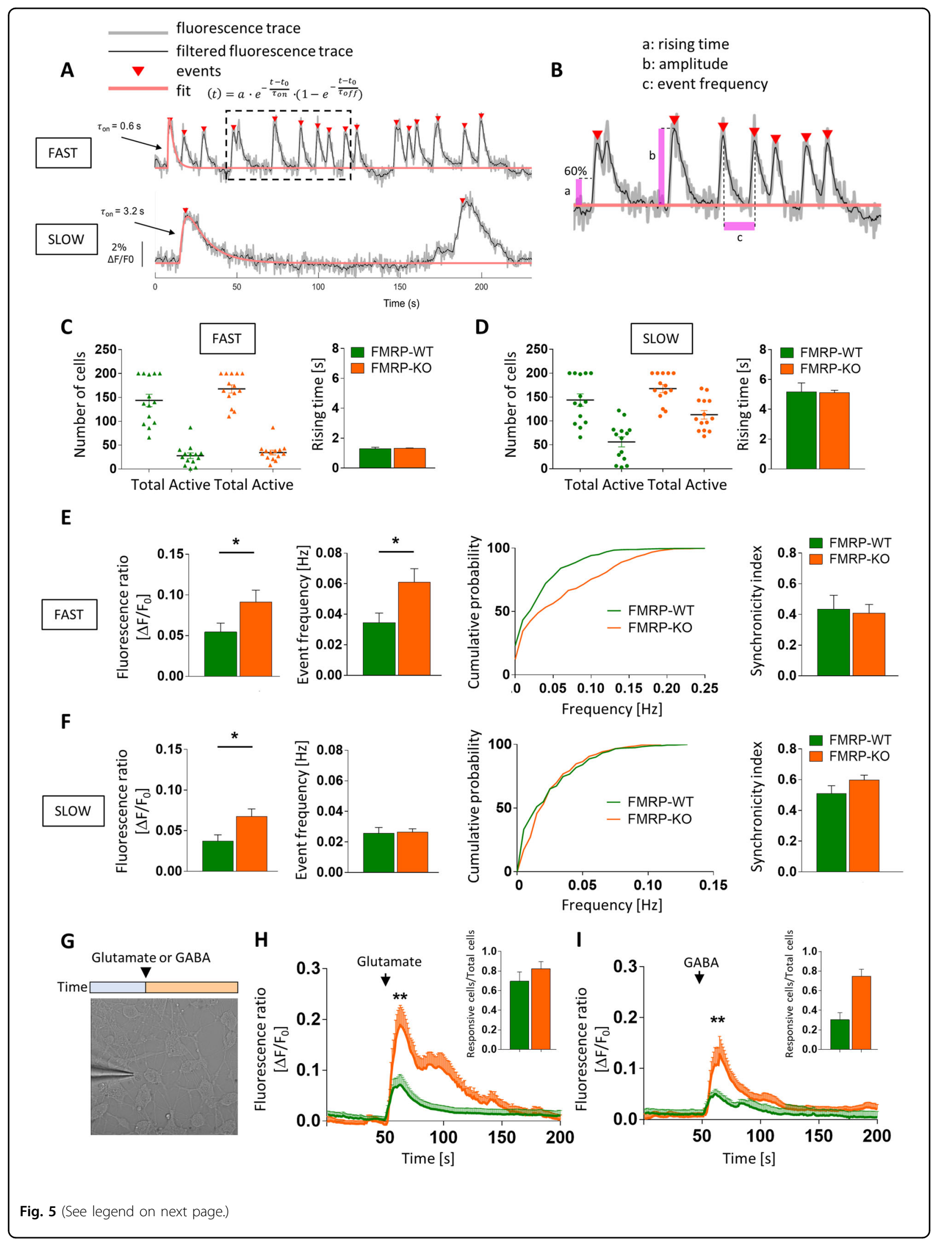




\begin{abstract}
(see figure on previous page)
Fig. 5 Spontaneous and evoked calcium activity analysis of 2D cortical cultures at day 70. A Calcium traces as a function of $\triangle F / F_{0}$ of FAST (top) and SLOW (bottom) active cells. B Representative calcium trace (insert from panel A) showing extracted functional properties: rising time (a), amplitude (b), and event frequency (c). C FAST active cells with respect to total cells in both FMRP-KO and FMRP-WT populations ( $n=14$ FOVs for genotype; $144 \pm 13$ total cells in WT, $28 \pm 6$ active cells in WT; $168 \pm 8$ total cells in KO, $34 \pm 5$ active cells in $\mathrm{KO}$; rising time $1.3 \pm 0.1$ sec in WT and $1.3 \pm 0.03 \mathrm{sec}$ in $\mathrm{KO}$; differentiation batches $=1$ ). D SLOW active cells with respect to total cells in both FMRP-KO and FMRP-WT populations ( $n=14$ FOVs for genotype; $144 \pm 13$ total cells in WT, $56 \pm 11$ active cells in WT; $168 \pm 8$ total cells in KO, $113 \pm 9$ active cells in KO; rising time $5.2 \pm 0.6$ sec in $W T$ and $5.1 \pm 0.17 \mathrm{sec}$ in $\mathrm{KO}$; differentiation batches =1). $\mathbf{E}$ Graph plots reporting the functional properties of FAST active cells: amplitude (left, $n=14$ FOVs, ${ }^{*} p<0.05 \mathrm{KO}$ vs WT, MW test), frequency and relative cumulative distribution (middle, $n=14 \mathrm{FOVs}, 0.035 \mathrm{~Hz}$ in WT and $0.06 \mathrm{~Hz}$ in $\mathrm{KO},{ }^{*} p<0.05$ KO vs WT) and synchronicity (right) of spontaneous calcium events of FMRP-WT and FMRP-KO fast cells $(n=14,0.43 \pm 0.09$ in WT and $0.41 \pm 0.06$ in $\mathrm{KO}$ not significant). $\mathbf{F}$ Graph plots reporting the functional properties of SLOW active cells amplitude (left, $n=14 \mathrm{FOVs}$, ${ }^{*} p<0.05 \mathrm{KO}$ vs WT, MW test), frequency and relative cumulative distribution (middle, $n=14 \mathrm{FOVs}, 0.025 \mathrm{~Hz}$ in WT and $0.025 \mathrm{~Hz}$ in KO, not significant) and synchronicity (right) of spontaneous calcium events of FMRP-WT and FMRP-KO slow cells ( $n=14,0.51 \pm 0.05$ in WT and $0.6 \pm 0.03$ in KO, not significant). G Representative FOV showing a puffer pipette for local Glutamate and GABA application with a cartoon showing the experimental approach. $\mathbf{H}$ Glutamatergic calcium transient response of FMRP-KO and FMRP-WT cortical neurons after puffer applications ( $n=5$ FOVs, $23 \pm 4.7$ total cells and $17 \pm 4$ active cells in WT; $n=4$ FOVs, $22 \pm 2$ total cells and $18 \pm 1$ active cells in $\mathrm{KO} ;{ }^{* *} p<0.01 \mathrm{KO}$ vs WT; differentiation batches $=1$ ). I GABAergic calcium transient response of FMRP-KO and FMRP-WT cortical neurons after puffer applications ( $n=5 \mathrm{FOVs}, 22.6 \pm 3.5$ total cells and $7 \pm 1.8$ active cells in WT; $n=5$ FOVs, $14.6 \pm 1.7$ total cells and $11.4 \pm 2$ active cells in $\mathrm{KO},{ }^{* *} p<0.01 \mathrm{KO}$ vs WT; differentiation batches $=1$ ).
\end{abstract}

\section{Development of a brain organoid-based FXS 3D disease model}

The next step was to improve the hiPSC-based FXS model moving to a more complex, physiologically relevant in vitro model of the disease such as brain organoids.

The timeline of the experimental strategy used to generate self-assembled brain organoids from FMRP-WT and FMRP-KO hiPSCs is reported in Supplementary Fig. S2B. During organoids maturation, at day 50 we observed the appearance of markers typical of the ventricular and subventricular zones as N-CADHERIN (Fig. 6A), SOX2 (Fig. 6B), FOXG1, and PAX6 (Fig. 6C) confirming the presence of a germinal layer; moreover, developing cortical plates were positive for deep layer cortical neurons as revealed by the presence of CTIP2, TBR1, and MAP2 positive cells (Fig. 6D, Supplementary Fig. S6A, and higher magnification insert in Supplementary Fig. S6B), suggesting the initial formation of the cortical stratification. At day 100, the positive staining for CTIP2, PAX6, and GFAP revealed the presence of neuronal and glial cells, validating our protocol for cortical organoids generation (Fig. 6E, and Supplementary Fig. S6C for higher magnification).

As the FMRP deficiency is believed to alter the early developmental stages of the corticogenesis, we compared cortical organoids obtained from FMRP-WT and FMRP$\mathrm{KO}$ iPSCs at morphological and cellular level. After 70 days in culture, brain organoids displayed typical laminar structures, as represented in Fig. 7A, left. We observed that at this stage FMRP-KO organoids were significantly bigger in size than the FMRP-WT ones. Indeed, FMRP-KO organoids displayed a significantly higher number of cortical plates (Fig. 7A, right). Consistently, more general parameters, as maximun organoid diameter (Fig. 7C), surface area, (Fig. 7D), and perimeter (Fig. 7E) were higher in FMRP-KO organoids respect to FMRP-WT.
Moreover, the characterization of the cellular identity within the cortical plates indicated that while the staining of the neuronal markers MAP2 and for TUJ1 was similar in the two genotypes (Fig. 8A, B, and Supplementary Fig. S7A, S7B), FMRP-KO cerebral organoids displayed increased GFAP labeling (Fig. 8A, B), reminiscent to what was observed in 2D cultures (Figs. 2D and 4A). This was assessed both by the measure of GFAP signal intensity and by counting the number of GFAP positive cells (Fig. 8C, D). At day 100 CTIP2 positive staining revealed the presence of layer $\mathrm{V}$ cortical neurons in both FMRPWT and KO organoids (Supplementary Fig. S6C).

To further characterize the maturation of cerebral organoids we analyzed the cellular identity within the cortical plates of FMRP-WT and FMRP-KO organoids at day 100 using MAP2 and TUJ1 as neuronal markers and GFAP as astrocytic marker (Fig. 9A, and Supplementary Fig. S7). We found that following the corticogenesis, at day 100, FMRP-KO organoids were characterized by increased GFAP staining and unaltered MAP2 staining (Fig. 9A, B), assessed as fluorescence intensity (Fig. 9C, and Supplementary Fig. S7C, S7D). The number of astrocytes, evaluated as GFAP positive cells, at day 100 displayed a tendency to be higher in FMRP-KO organoids compared to WT (Fig. 9D). These data suggest that a FXS phenotype can be obtained in FMRP-KO 3D selfassembled brain organoids that, with respect to isogenic parental FMRP-WT line, display increased number of cortical plates, bigger size, and accelerated maturation of GFAP-positive astrocytes.

\section{Discussion}

The development of effective drugs for FXS is challenging because of the limited understanding of its pathophysiology, the differences between patients and animal models, and the difficulties in modeling FXS in vitro and in vivo. 


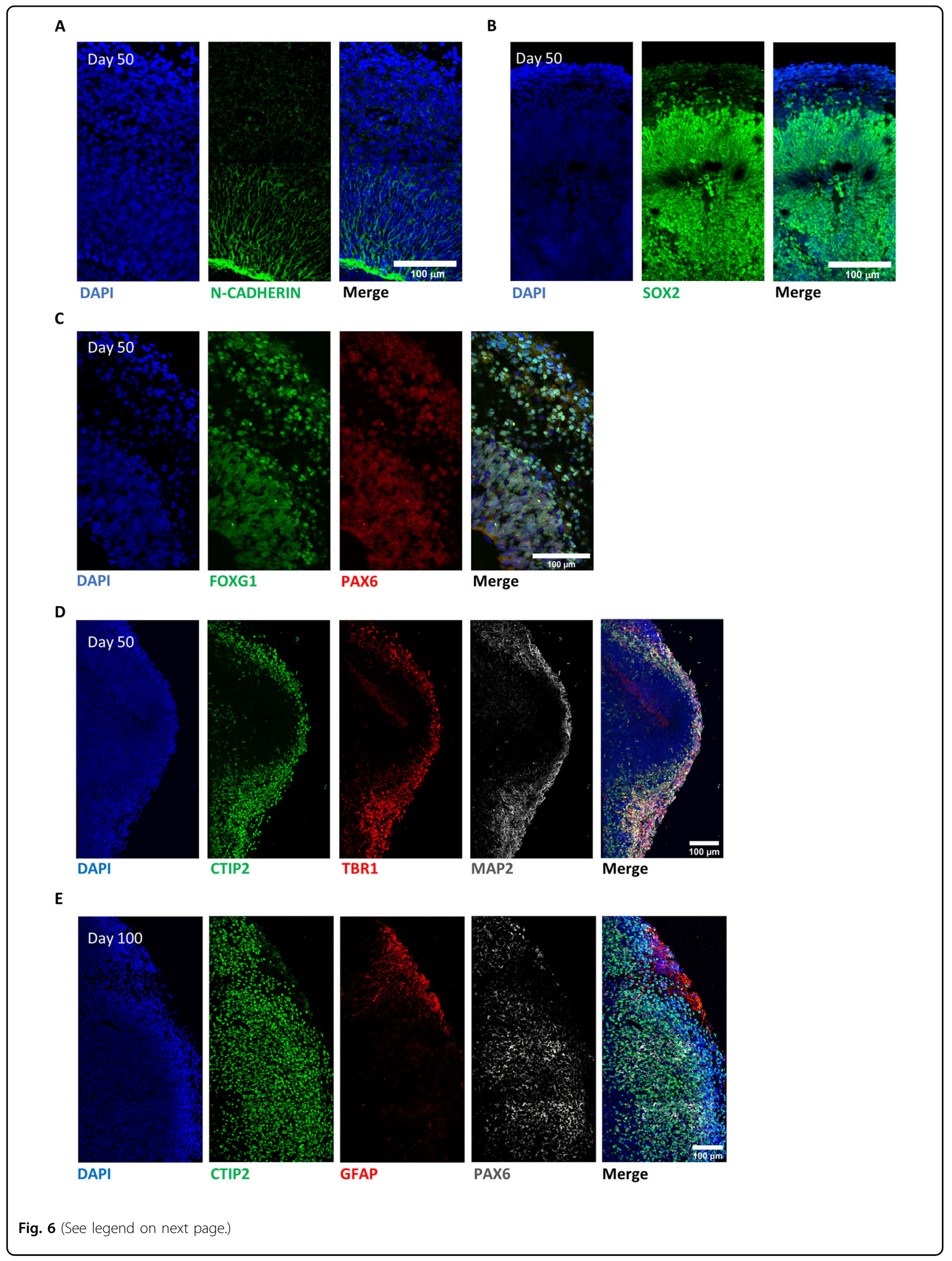


(see figure on previous page)

Fig. 6 Evaluation of cellular identity and morphology in 3D brain organoid. A Representative immunostaining showing the presence of a NCADHERIN-positive (green) germinal layer in correspondence of the apical membrane of the ventricular zone layer in a FMRP-WT brain organoid at day 50. B, C Representative images revealing forebrain progenitor markers in developing cortical plates within FMRP-WT brain organoid at day 50: B SOX2 (green), DAPI (blue); C PAX6 (red), FOXG1 (green) and DAPI (blue). D Representative staining of early-born deep-layer cortical neurons positive for TBR1 (red), CTIP2 (green) markers, and pan-neuronal MAP2 marker (white) in a FMRP-WT brain organoid at day 50. E Representative images of a cortical plate from a FMRP-WT brain organoid at day 100 revealing heterogeneous cellular population, labeled for specific cortical neuronal marker (CTIP2, green), astrocytic marker (GFAP, red), and neuronal progenitor marker (PAX6, white). Nuclei were stained with DAPI (blue). Scale bar: 100 um.

Progresses in disease-relevant hiPSC generation and modification by genome editing and in the generation of $3 \mathrm{D}$ organoids provide novel possibilities for disease modeling and drug screening for FXS. This study reports the generation of 2D and 3D in vitro FXS models from control and isogenic FMRP-KO hiPSCs, which recapitulate in vitro relevant FXS phenotypes. The long-term aim is to overcome the issues due to differences between human patients and animal models. Indeed, a proper in vitro human disease model in which FXS evidences could be confirmed and implemented is still missing. Here we show that both control and FMRP-KO hiPSCs can be converted to functionally mature cortical neurons and cerebral organoids, and that these in vitro systems display, up to 100 days in vitro, a FXS phenotype and can be considered promising disease models to study FXS and to develop and test disease-modifying drugs. Specifically, using an isogenic FMRP-KO hiPSC line we obtained a 2D mixed cortical culture displaying: (i) altered neuronal and glial gene expression with increased expression of the astrocyte marker GFAP; (ii) increased network activity; (iii) increased excitation/inhibition ratio. Moreover we report for the first time the generation of a 3D brain organoid system to model FXS, characterized by: (i) increased size and number of cortical plates, (ii) unaltered neuronal staining, and (iii) increased GFAP immunoreactivity.

In our study, the time-series analysis revealed that the lack of FMRP increased the expression of some neural progenitor genes at day 10. However, such upregulation was modest, statistically significant only for $S O X 2$, and transient, suggesting that absence of FMRP did not lead to substantial alteration of the neural progenitor population. Conversely, we consistently observed increased levels of the astrocyte marker GFAP, with concomitant decrease of the neuronal precursor marker TBR2, at day 25. Hence, we speculate that FMRP is responsible for the correct balance and development of neuronal and glial components in cerebral cortex, in line with what was observed in human neural progenitor cells ${ }^{36}$ and in Fmr1 KO mouse model $^{37}$.

One important point of strength of our system is that, differently to what was observed in murine Fmr1 KO cortical neurons ${ }^{38}$, hiPSC derived FMRP-KO cultures are characterized by a gain of function of spontaneous network activity even in the absence of specific challenging protocols. Indeed, in control culture condition we observed that the silencing of FMRP induced a significant increase of both amplitude and frequency of "fast", neuronal, spontaneous calcium events, and a significant increase of the amplitude of "slow" glial events.

Notably, together with the enhancement of network activity we report a different pattern of response to focal application of both glutamate and GABA in FMRP-KO cortical neurons, pointing to an unbalance of excitation and inhibition that relies on both systems. Indeed, while in our culture the large majority of cells responded to focal glutamate application with a fast intracellular calcium rise in both genotypes, the amplitude of glutamatergic evoked calcium transients was significantly higher in FMRP-KO neurons with respect to FMRP-WT. This phenomenon can be ascribed either to a functional upregulation of glutamatergic receptors, or to an increased depolarizing response, as reported for mouse neuronal progenitors lacking FMRP ${ }^{39,40}$. Moreover, although at day 70 the number of glutamatergic synapses was similar in FMRP-WT and FMRP-KO cortical cultures, as revealed by the pre (VGLUT1) and post-synaptic (PSD95) staining, we cannot exclude a different contribution of ionotropic and metabotropic glutamate receptors between the two genotypes in mediating the neuronal depolarization that might contribute to glutamate-induced calcium transients, as in murine neuronal progenitors ${ }^{41}$.

Strikingly, we report here that in FMRP-KO culture the focal application of GABA induced a depolarizing response, as revealed by the appearance of a calcium transient evoked by GABA puff. This phenomenon may rely on an immature phenotype of FMRP-KO hiPSCderived cortical neurons which could be characterized by a depolarized chloride equilibrium. Indeed, GABA, the major inhibitory neurotransmitter in the brain, binds to GABA-A (ionotropic) or GABA-B (metabotropic) receptors that in mature healthy neurons mediate an inhibitory response that leads to membrane hyperpolarization. GABA transmission plays indeed a key role in setting the excitation/inhibition ratio, which is abnormal in FXS, as well as in autism and other neurodevelopmental disorders $^{42}$. Moreover, even if many studies into GABA- 
A FMRP-WT (3D) d70

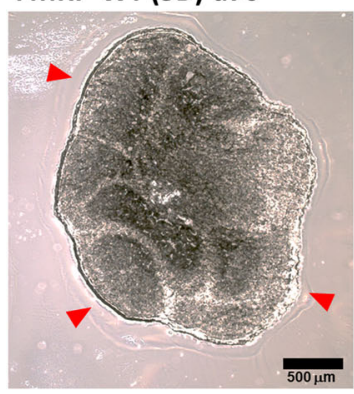

B

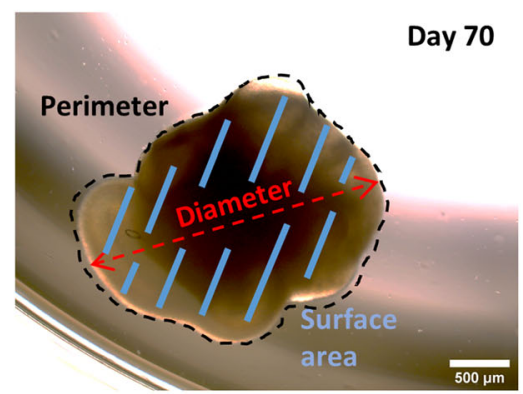

D

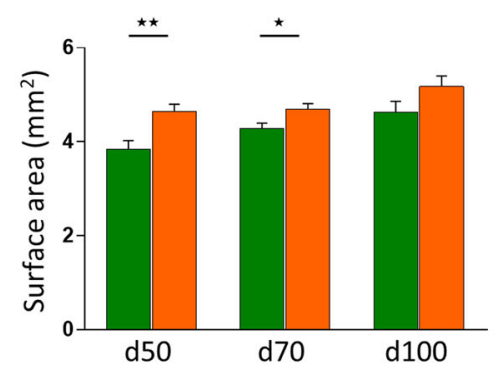

FMRP-KO (3D) d70

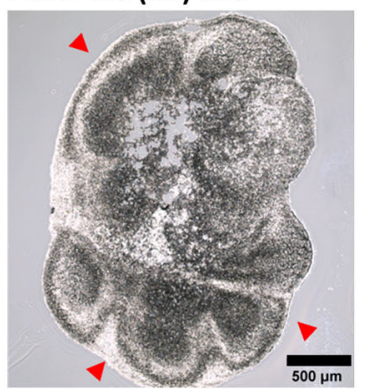

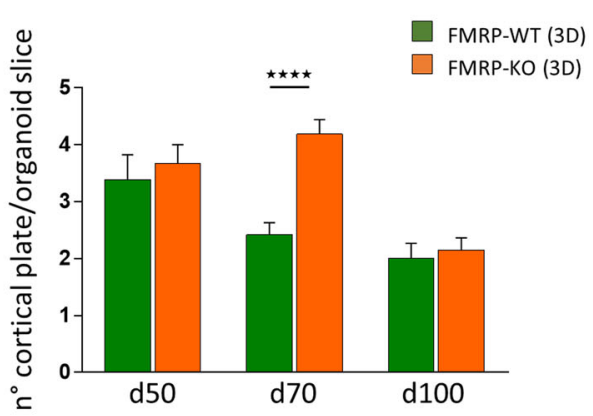

C
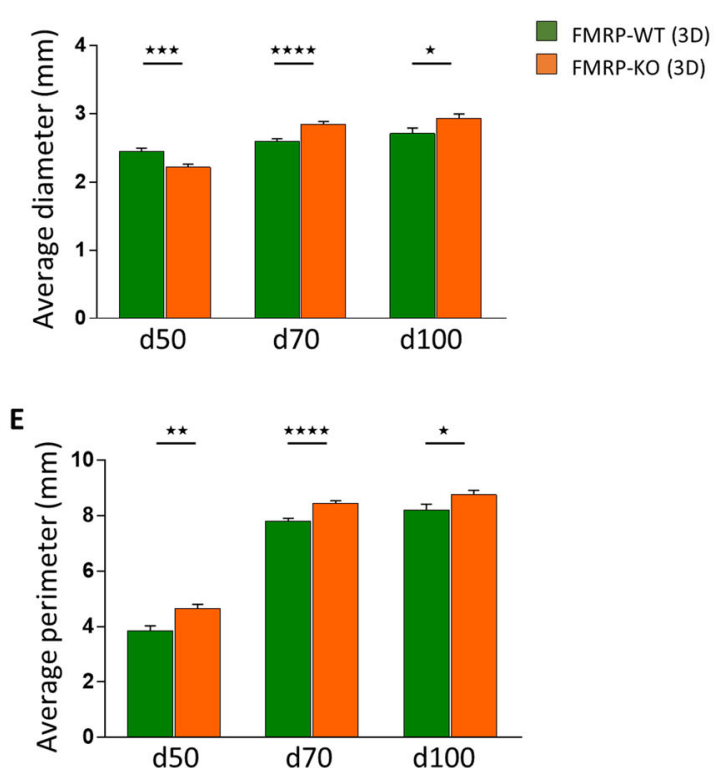

Fig. 7 Quantification of organoid size parameters at different time points. A Left: Representative slices of FMRP-WT and FMRP-KO cryOsectioned brain organoids at day 70 indicating the presence of developing cortical plates (arrows) within the organoids. Right: Bar chart showing the quantification of cortical plates within FMRP-WT and FMRP-KO brain organoids at day 50 (WT 3.4 $\pm 0.5, \mathrm{KO} 3.6 \pm 0.3, p=0.61, \mathrm{MW}$ test; $n=6 / 1 \mathrm{WT}$, $n=5 / 1 \mathrm{KO}$ organoids/batches), at day 70 (WT 2.4 $\pm 0.2, \mathrm{KO} 4.2 \pm 0.3,{ }^{* * *} p<0.0001$, MW test; $n=12 / 3 \mathrm{WT}, n=14 / 2 \mathrm{KO}$ organoids/batches) and at day 100 (WT 2 $\pm 0.3, \mathrm{KO} 2.1 \pm 0.2, p=0.72$, MW test; $n=8 / 1 \mathrm{WT}, n=9 / 1 \mathrm{KO}$ organoids/batches). B Representative tracing of organoid maximum diameter, perimeter, and surface area at day 70. The quantification of the organoid size parameters was performed at day 50, 70 , and 100 . $\mathbf{C}$ Bar chart showing organoid diameter (day $50{ }^{* * *} p<0.001$ : WT $n=48 / 1, \mathrm{KO} n=41 / 1$ organoids/batches; day $70{ }^{* * * *} p<0.0001: \mathrm{WT} n=110 / 3, \mathrm{KO} n=102 / 2$ organoids/batches; day $100 * p<0.05$ : WT $n=21 / 1, \mathrm{KO} n=22 / 1$ organoids/batches. D Bar chart showing organoid surface area (day $50 * * p<0.01$; day $70{ }^{*} p<0.05$; day $100, p=0.1 ; n$ as in C). E Bar chart showing organoid perimeter (day $50{ }^{* *} p<0.01$; day $70{ }^{* * *} p<0.0001$; day 100 , ${ }^{*} p<0.05 ; n$ as in C).

mediated mechanisms in FXS indicate a reduction in both GABA-A and GABA-B activation in Fmr1-KO mice ${ }^{43-45}$, and in embryonic stem cell-derived human FXS neurons $^{46}$, GABA-A and GABA-B agonists that have been tried in clinical trials, failed in reducing cortical hyperexcitability ${ }^{47}$.

We can speculate that this failure relies on the delayed developmental switch of chloride equilibrium in FXS; indeed, when the equilibrium potential for chloride is depolarized, GABA mediated responses depolarize neurons, increasing their excitability and triggering spiking and calcium entry into neurons as during development and in several pathological conditions ${ }^{48-50}$. Indeed, a delay in the switch of the chloride reversal potential has been reported in cortical neurons of Fmr1 KO mice, causing the change in GABA transmission polarity, which in turn might affect neuronal development in the cortex of Fmr1 KO mice ${ }^{51}$. In summary, we can speculate that, in FMRP-KO coltures, besides the unbalance of glutamatergic and GABAergic systems, the upregulation of astrocytes may contribute to the enhancement of network activity $^{52}$. 


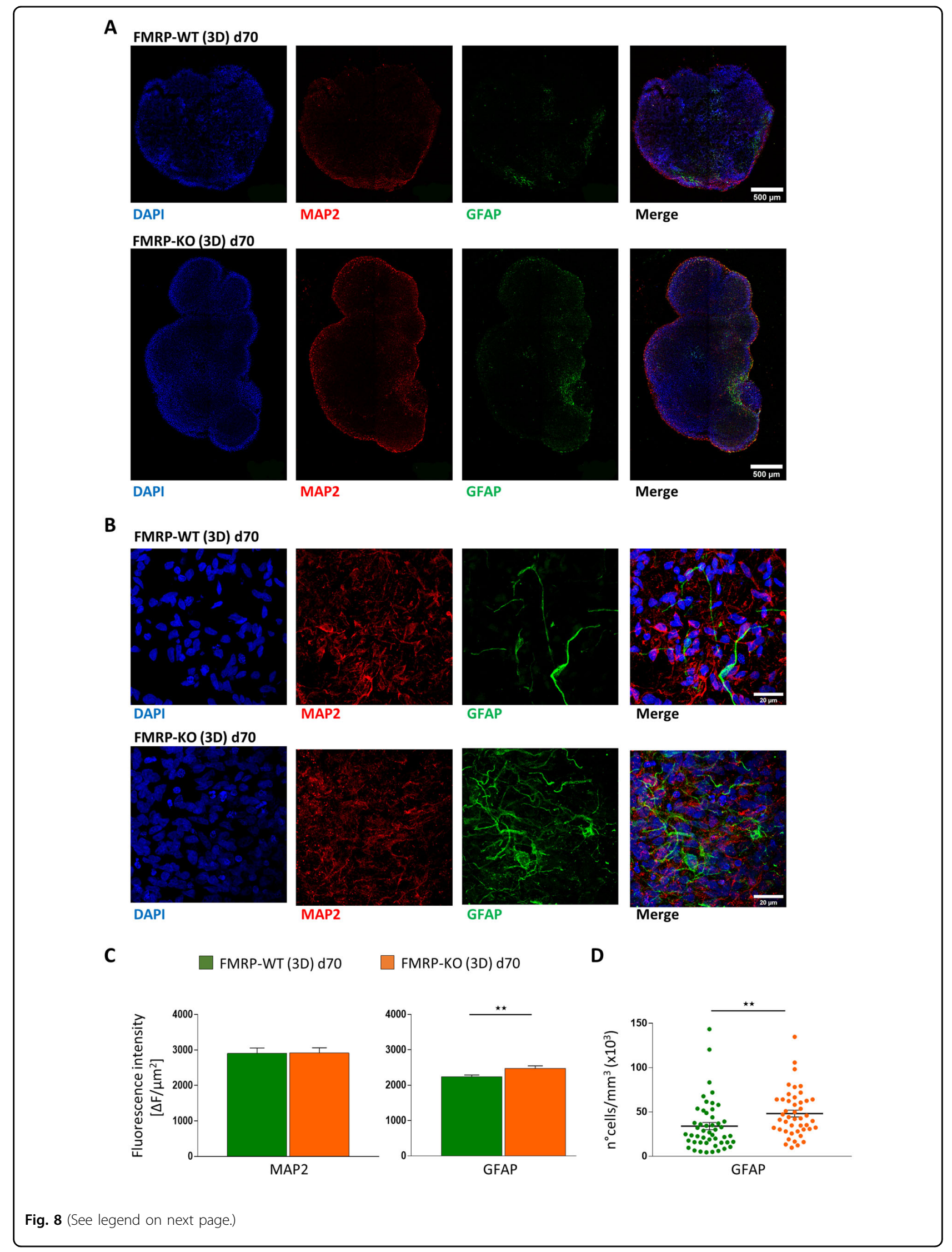


(see figure on previous page)

Fig. 8 Immunofluorescence analysis of 3D brain organoids at day 70. A Cellular identity characterization in FMRP-WT (top) and FMRP-KO (bottom) brain organoids. Immunostaining at day 70 revealed the presence of cells positive for pan-neuronal marker MAP2 (red) and GFAP expressing astrocytes (green). Nuclei were stained with DAPI (blue). Scale bar: $500 \mu \mathrm{m}$. B Higher magnification of representative GFAP-positive regions within FMRP-WT (top) and FMRP-KO (bottom) brain organoids at day 70 . Scale bar: $20 \mu \mathrm{m}$. C Bar charts showing the fluorescence intensity quantification of MAP2 signal ( $p=0.84$, MW test) and GFAP signals (** $p<0.01 ; \mathrm{WT} n=42 / 7 / 2, \mathrm{KO} n=29 / 6 / 2$, slices/organoids/batches). D Scatter plot of astrocyte density in FMRP-WT ( $n=49 / 18 / 8 / 2$, FOVs/slices/organoids/batches) and FMRP-KO brain organoids $(n=45 / 11 / 5 / 2$, FOVs/slices/ organoids/batches) at day $70\left({ }^{* *} p<0.01, \mathrm{MW}\right.$ test).

By taking advantage of hiPSC-derived cerebral organoids to model the complexity of human brain development, we were able to recapitulate some features of FXS. We found, in 3D cortical organoids derived from FMRPKO hiPSCs, a significant increase in cortical organoid size, with similar expression of neuronal marker MAP2 and an increase in astrocyte reactivity at different time points. The increase in GFAP signal and in the number of GFAP positive cells was observed also in the $2 \mathrm{D}$ neural differentiation system, can account for the increased organoid size, and resembles what was reported in primary glial cells isolated from Fmr1 KO mice ${ }^{37}$. Astrocytes are well recognized as active players in the neurobiology of FXS and involved in the correct development and maturation of hippocampal synapses ${ }^{53-55}$. The presence of neuronal and glial cells in our FMRP-KO hiPSC-derived 2D cultures and 3D organoids will thus provide an important tool for deepening our knowledge on astrocyte biology in FXS during the early stage of development.

We speculate that, in this novel 2D and 3D iPSC-based models of human cortex, FMRP is responsible for the correct balance of neuron and glial development. This could be mediated, similarly to what was observed in murine models, by the overexpression of GSK- $3 \beta^{56}$ that, positively regulating Notch pathway ${ }^{57}$, has been found to be sufficient to initiate a rapid and irreversible loss of neurogenesis by accelerating gliogenesis ${ }^{58}$. Thus we suggest that the 3D human brain organoid could efficiently recapitulate the in vivo pathological phenotypes.

In summary, we used the $3 \mathrm{D}$ organoid platform to recapitulate the development of cortical phenotype of FXS. This experimental platform can be potentially applied for FXS in vitro modeling and exploring effective treatments for this neurodevelopmental disorder.

\section{Materials and methods}

\section{Generation of the FMRP-KO hiPSC line}

The pGEM-T donor vector (Promega) was engineered by introducing a donor construct, consisting of a cassette encoding for the self-cleavage peptide T2A, the puromycin resistance gene (PURO-resistance), and the beta$\mathrm{GH}$ cleavage and polyadenylation site $(\mathrm{pA})$. The cassette was amplified from AAVS1-Hb9-GFP vector ${ }^{59}$ and flanked by FMR1 homology sequences to promote its integration in the genome upon homology-directed repair, interrupting the FMR1 coding sequence. A second vector, pX330-U6-Chimeric_BB-CBh-hSpCas9 (Addgene, ID \#42230), coding for the nuclease Cas9 and the chimeric guide RNA (gRNA), was engineered inserting guide sequences targeting FMR1 exon 2 (gRNA\#1 Fw CACCGGTCCAGTTTGAGTGCTTTTC / Rv: AAACGA AAAGCACTCAAACTGGACC; gRNA\#2 Fw: CACCGT TCCTGAAAAGCACTCAAAC / Rv: AAACGTTTGAG TGCTTTTCAGGAAC).

Cells were electroporated with $2 \mu \mathrm{g}$ of donor construct and $3 \mu \mathrm{g}$ of px330-Cas9 plasmid using a Neon Transfection System (Thermo Fisher Scientific) and the following settings: $1200 \mathrm{~V}, 30 \mathrm{~ms}, 1$ pulse. After transfection, hiPSCs were cultured in presence of $10 \mu \mathrm{M}$ ROCK inhibitor (Y27632; Enzo Life Science) for one day. Clonal selection was carried out with $0.5 \mu \mathrm{g} / \mathrm{ml}$ puromycin for 15 days, and surviving clones were individually passaged and characterized as described in Fig. 1A, B.

\section{Human iPSC maintenance and differentiation into cortical neurons}

FMRP-WT and FMRP-KO hiPSC lines were cultured in Nutristem-XF (Biological Industries) supplemented with 0.1\% Penicillin-Streptomycin (Thermo Fisher Scientific) onto hESC-qualified Matrigel (CORNING) functionalized plates. The culture medium was refreshed every day and cells were passaged every $4-5$ days using $1 \mathrm{mg} / \mathrm{mL}$ Dispase II (Thermo Fisher Scientific). Cells were routinely tested for mycoplasma contamination.

hiPSCs were differentiated into cortical neurons according to a previously published protocol ${ }^{33}$ with minor modifications. Briefly, hiPSCs were treated with Accutase (Thermo Fisher Scientific) and the single-cell suspension was plated in Matrigel-coated dishes at a seeding density of 100,000 cells per $\mathrm{cm}^{2}$, in Nutristem-XF supplemented with $10 \mu \mathrm{M}$ ROCK inhibitor. The day after seeding the medium was changed to N2B27 medium consisting of DMEM-F12, Dulbecco's Modified Eagle's Medium/ Nutrient Mixture F-12 Ham (Sigma Aldrich), Neurobasal Medium (Thermo Fisher Scientific), 1X N2 supplement (Thermo Fisher Scientific), 1X GlutaMAX (Thermo Fisher Scientific), 1X MEM-NEAA (Thermo Fisher Scientific), 1X B27 (Thermo Fisher Scientific), 1X Penicillin- 

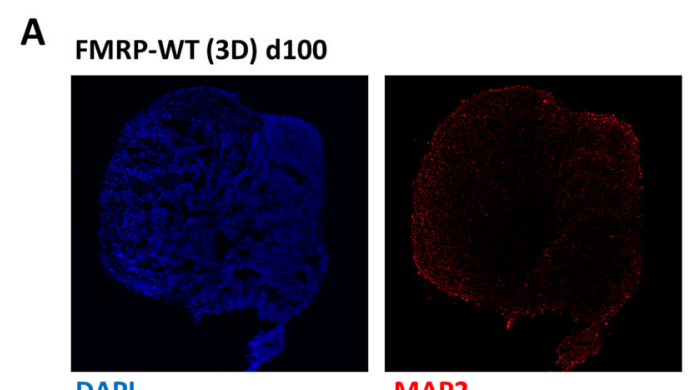

MAP2
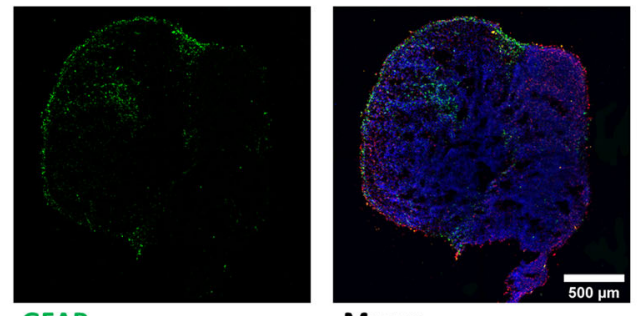

FMRP-KO (3D) d100
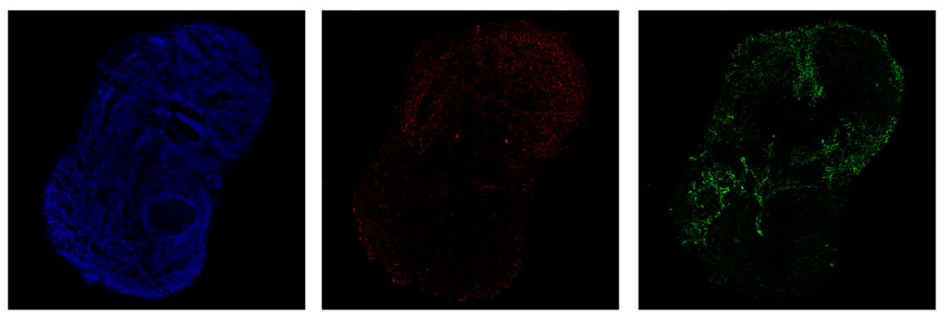

Merge

DAPI

MAP2

GFAP

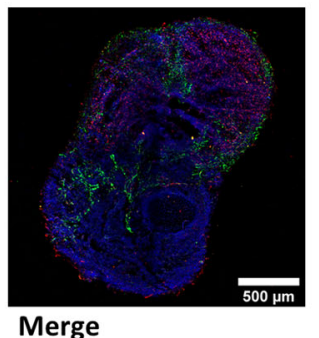

B

FMRP-WT (3D) d 100
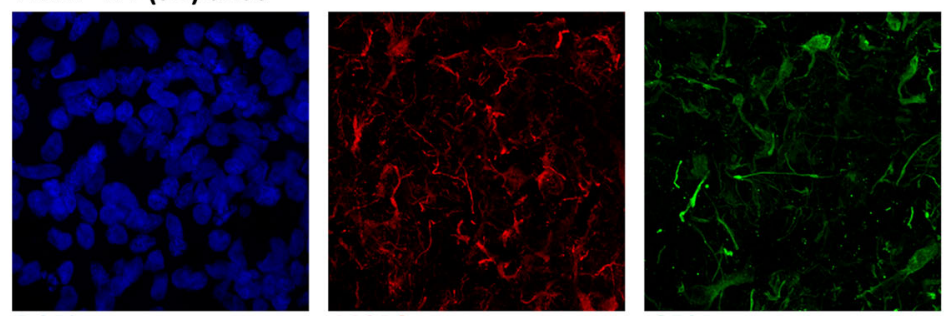

MAP2

GFAP

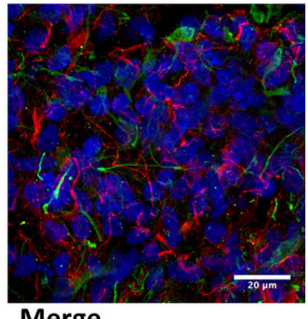

FMRP-KO (3D) d100
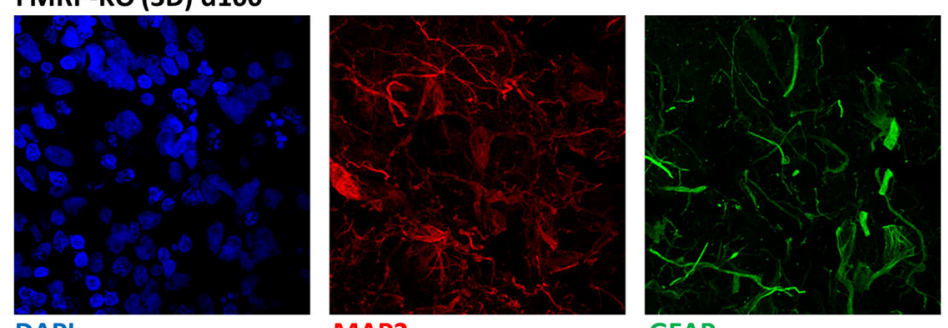

Merge

$$
\text { DAPI }
$$

MAP2

GFAP

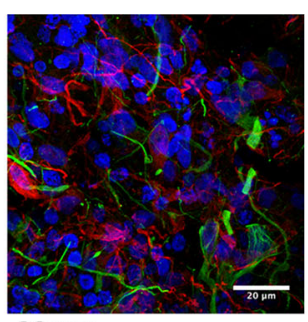

C

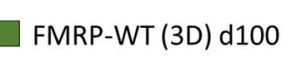

$\square$ FMRP-KO (3D) d100

D
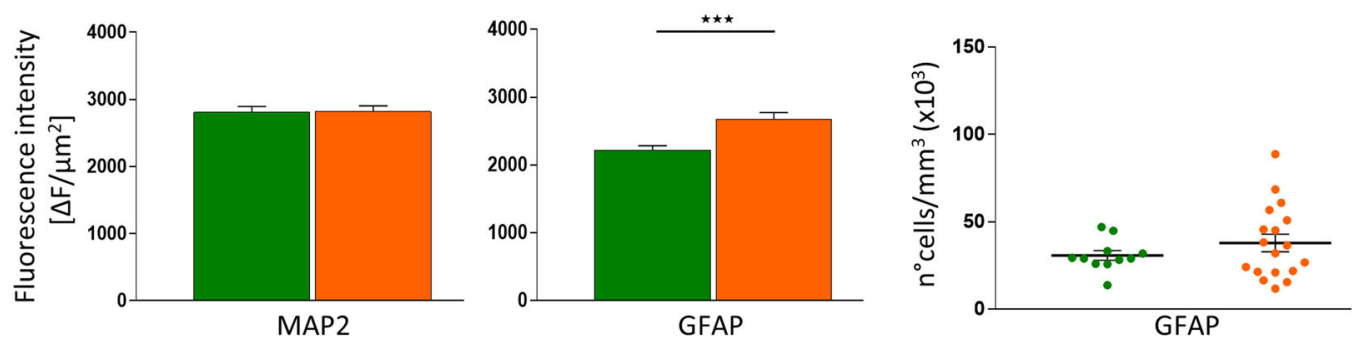

Fig. 9 (See legend on next page.) 
(see figure on previous page)

Fig. 9 Immunofluorescence analysis of 3D brain organoids at day 100. A Cellular identity characterization in FMRP-WT (top) and FMRP-KO (bottom) brain organoids. Immunostaining at day 100 revealed the presence of cells positive for pan-neuronal marker MAP2 (red) and GFAP expressing astrocytes (green). Nuclei were stained with DAPI (blue). Scale bar: $500 \mu \mathrm{m}$. B Higher magnification of representative GFAP-positive regions within FMRP-WT (top) and FMRP-KO (bottom) brain organoids at day 100. Scale bar: $20 \mu \mathrm{m}$. C Bar charts showing the fluorescence intensity quantification of MAP2 signal ( $p=0.9$, MW test) and GFAP signals ( ${ }^{* * *} p<0.001$; WT $n=24 / 4 / 1, \mathrm{KO} n=32 / 4 / 1$, slices/organoids/batches). D Scatter plot of astrocyte density in FMRP-WT $(n=55 / 6 / 4 / 1$, FOVs/slices/ organoids/batches) and FMRP-KO brain organoids $(n=58 / 5 / 4 / 1$, FOVs/slices/ organoids/batches) at day $100(p=0.3)$.

Streptomycin (Thermo Fisher Scientific). This was considered as day 0 (D0). At this stage, SMAD inhibitors, $10 \mu \mathrm{M}$ SB431542 and $500 \mathrm{nM}$ LDN-193189 (Cayman Chemical), were added daily to induce neural fate. After 10 days, the uniform neuroepithelial sheet was broken into clumps of approximately 500 cells/clump and replated onto $1 \mathrm{X}$ poly-L-ornithine/laminin (Sigma Aldrich) coated dishes in N2B27 medium. At day 20, cells were dissociated using Accutase and plated onto poly-L-ornithine/laminin-coated dishes at a density of 100,000 cells per $\mathrm{cm}^{2}$ in N2B27 medium supplemented with $10 \mu \mathrm{M}$ ROCK inhibitor for the first $24 \mathrm{~h}$. Culture medium was changed three times a week. At day 27, $2 \mu \mathrm{M}$ Cyclopamine (Merck Life Science) was freshly added to N2B27 medium for 4 days. At day 30 to 35, immature neurons were dissociated using Accutase and re-plated into polyL-ornithine/laminin-coated dishes at a density of 100,000 cells per $\mathrm{cm}^{2}$ in N2B27 medium supplemented with $20 \mathrm{ng} / \mathrm{mL}$ BDNF (Sigma Aldrich), $20 \mathrm{ng} / \mathrm{mL}$ GDNF (Peprotech), $20 \mathrm{ng} / \mathrm{mL}$ ascorbic acid (Sigma Aldrich), $1 \mathrm{mM}$ cyclic AMP (Sigma Aldrich), and $5 \mu \mathrm{M}$ DAPT (Adipogen Life Sciences).

\section{Generation of self-assembled brain organoids}

A slightly modified version of Lancaster's protocol ${ }^{60}$ was used for the generation of cerebral organoids. After removing any spontaneously differentiated cells, hiPSC colonies were dissociated into a single-cell suspension and resuspended at a density of 180 cells/ $\mu \mathrm{L}$ in Nutristem-XF with $10 \mu \mathrm{M}$ ROCK inhibitor. 9000 cells were then plated into each well of a low-attachment 96-well U-bottom plate (CORNING). After $48 \mathrm{~h}$, small embryoid bodies (EBs) of about 100-200 $\mu \mathrm{m}$ diameter were visible and the medium was switched to Neural Induction Medium (NIM) consisting of DMEM-F12 supplemented with $1 \mathrm{X}$ N2, 1X GlutaMAX supplement, 1X MEM-NEAA, and 1X penicillin-streptomycin, $1 \mu \mathrm{g} / \mathrm{mL}$ of Heparin (Sigma Aldrich). EBs were fed every other day, being careful not to disrupt them, until optically translucent edges appeared confirming the neuroepithelium formation. On day 15, EBs were encapsulated into Geltrex (Thermo Fisher Scientific) droplets and each spheroid was positioned in a single well of a low-attachment 24 well plate (CORNING) in Differentiation Neural Medium (DMEM-F12,
Neurobasal medium, 1X GlutaMAX supplement, 0.5X MEM-NEAA, $1 \mathrm{X}$ penicillin-streptomycin, 0.5X N2, and $0.5 \mathrm{X}$ B27 without vitamin A). After 4 days in static culture conditions, organoids were transferred to an orbital shaker (110 RPM), which facilitates the nutrient diffusion within the organoid, fed with the Maturation medium containing vitamin A (DMEM-F12, Neurobasal medium, 1X GlutaMAX supplement, 0.5X MEM-NEAA, 1X penicillin-streptomycin, $0.5 \mathrm{X} \mathrm{N} 2$ and $0.5 \mathrm{X}$ B27 with vitamin A). The culture medium was changed every 3-4 days. During the whole growing period, the organoids were grown separately, each in a single well, to avoid aggregation. Organoids with undefined borders and/or devoid of neuroepithelial structures were excluded from subsequent analyses.

\section{Brain organoids morphological analysis}

Brain organoids images were collected in light transmission using an EVOS microscope (Thermo Fisher Scientific) equipped with a $4 \mathrm{x}$ objective. Fiji software was used for calculating the organoid surface area (expressed in $\mathrm{mm}^{2}$ ) and perimeter and maximum diameter (expressed in $\mathrm{mm}$ ).

The number of cortical plates was evaluated at days 50/ $70 / 100$ on one cryosectioned slice per organoid acquired with transmitted light image at $4 \mathrm{x}$ magnification. For each organoid the selected slices was the one with the highest number of cortical plates. Blind manual counting of cortical plates was then independently performed by 3 operators on slices from FMRP-WT and FMRP-KO organoids.

\section{Immunostaining and image acquisition and analisys of 2D cultures}

Monolayer cortical networks were fixed in $4 \%$ paraformaldehyde (PFA; Sigma Aldrich) solution for $15 \mathrm{~min}$ at room temperature and washed twice with $1 \mathrm{X}$ phosphate buffer solution (PBS; Thermo Fisher Scientific). Fixed cells were permeabilized with PBS containing $0.2 \%$ Triton X-100 (Sigma Aldrich) for $15 \mathrm{~min}$ and blocked with a solution containing $0.2 \%$ Triton X-100 and 5\% Goat Serum (Sigma Aldrich) for $20 \mathrm{~min}$ at room temperature. Cells were then incubated overnight at $4{ }^{\circ} \mathrm{C}$ with primary antibodies at the following dilutions: mouse anti-PAX6 
(sc81649 Santa Cruz Biotechnology, 1:50), mouse antiGFAP (MAB360 Merck Millipore, 1:500), chicken antiMAP2 (ab5392 Abcam, 1:2000), rabbit anti- $\beta$-TUBULIN III (TUJ1) (T2200 Sigma Aldrich, 1:2000), rabbit antiFMRP (4317 Cell Signaling, 1:50), mouse anti-VGLUT1 (135303 Synaptic Systems, 1:250), rabbit anti-PSD95 (3450 Cell Signaling, 1:250), mouse anti-GAD67 (sc28376 Santa Cruz, 1:250). For double immunostaining of S100 $\beta$ and GFAP, the ready-to-use polyclonal rabbit antiS100b antibody (Dako, Clone Z0311) was diluted 1:1 with blocking solution (0.2\% Triton X-100 and 3\%BSA) and used in combination with mouse anti-GFAP antibody (MAB360 Merck Millipore, 1:500). The day after, the primary antibody solution was washed out and cells were incubated with secondary antibodies for $1 \mathrm{~h}$ at room temperature in the dark. AlexaFluor secondary antibodies (Thermo Fisher Scientific) were used at the concentration of 1:750 (Supplementary Table 1), and DAPI (Sigma Aldrich) was used for nuclei staining.

For synapses quantification of 2D cortical networks, fluorescence images were acquired on an Olympus iX73 microscope equipped with an X-Light V3 spinning disc head (Crest Optics), an LDI laser illuminator (89 North), an Evolve EMCCD camera (Photometrics), and a MetaMorph software (Molecular Devices). Images of $512 \times 512$ pixels $(267 \times 267 \mathrm{~nm} /$ pixel $)$ were acquired with a 60x/NA 1.35 oil objective (Olympus) in stack with $\mathrm{z}$-step of $0.5 \mu \mathrm{m}$. The specificity of the staining was tested performing control experiments in the absence of primary antibody incubation. Some samples were incubated with antibody dilution buffer without primary antibodies to be confident that the synaptic signals were not confounded with any non-specific binding of secondary antibody in the culture sample. Quantification was performed on Fiji software using the "Puncta Analyzer" plugin. Stack images were flattened in a maximum intensity Z-projection and after removing the background noise, images were binarized allowing the count of synaptic puncta and the quantification of the colocalizing punctate. Specifically, as required by the plugin, the merged image representing presynaptic staining of VGLUT (channel 1) and postsynaptic protein PSD95 (channel 2) was splitted for threshold adjustment of each synaptic marker. The threshold was set to eliminate background unspecific signal for each channel. Finally, the "Puncta Analyzer" plugin performed quantification of synaptic puncta for each channel, and for the colocalized ones.

For astrocytes quantification on 2D cortical populations, images were analyzed on Fiji software quantifying the GFAP signal as fluorescence intensity. The fluorescence threshold was adjusted to accurately represent the number of GFAP-positive cell processes. Integrated density, mean background value, and the total area of the field of view (FOV) were calculated in order to get the mean fluorescence value per $\mu \mathrm{m}^{2}$.

[Integrated density $-($ FOV area $\times$ mean background value $)] /$ FOV area

\section{Immunostaining and image acquisition and analisys of brain organoid}

Brain organoids were collected at days 50, 70, and 100, incubated in 4\% PFA solution for $30 \mathrm{~min}$ and then moved in PBS with $30 \%$ sucrose overnight at $4{ }^{\circ} \mathrm{C}$. The following morning, the sucrose solution was removed and organoids were cryosectioned using a standard cryostat (Leica CM1860, Leica Biosystems). $50 \mu \mathrm{m}$ thick sections were collected on Ultra Plus slides (Thermo Fisher Scientific) and stored at $+4{ }^{\circ} \mathrm{C}$.

For immunostaining, organoid sections were quickly washed for $10 \mathrm{~min}$ in PBS and then incubated for $30 \mathrm{~min}$ with a warm antigen retrieval solution containing $1 \mathrm{X}$ Citrate buffer, at pH 6.0 (Sigma Aldrich). Samples were exposed to a $200 \mathrm{mM}$ Glycine (Sigma Aldrich) solution in PBS for 15 min before permeabilization in $0.3 \%$ Triton-X in PBS for $45 \mathrm{~min}$. After a double washing, sections were blocked in $0.3 \%$ Triton-X and 5\% goat serum in PBS for $1 \mathrm{~h}$. Tissues were then incubated overnight with primary antibodies in $0.3 \%$ Triton- $\mathrm{X}$ and $5 \%$ goat serum in PBS at the following dilutions: mouse anti-PAX6 (sc81649 Santa Cruz Biotechnology, 1:50), rabbit anti-PAX6 (HPA030775 Sigma Aldrich, 1:50), rabbit anti-FOXG1 (ab18259 Abcam, 1:200), mouse anti-GFAP (MAB360 Merck Millipore, 1:500), chicken anti-MAP2 (ab5392 Abcam, 1:1000), rabbit anti-N-CADHERIN (ab18203 Abcam, 1:50), rabbit anti-TBR1 (20932-1-AP Proteintech, 1:150), rabbit anti- $\beta$-TUBULIN III (TUJ1) (T2200 Sigma Aldrich, 1:1000), rat anti-CTIP2 (ab18465 Abcam, 1:300), mouse anti-SOX2 (MA1-014 Thermo Fisher Scientific, 1:200). The day after, the primary antibody solution was washed out and cells were incubated with secondary antibodies for $2 \mathrm{~h}$ at room temperature in a dark room. AlexaFluor secondary antibodies (Thermo Fisher Scientific) were used at the concentration of 1:750 (Supplementary Table 1), and Hoechst (Sigma Aldrich, 1:1000) was used for nuclei staining. Finally, after 3 washes with $0.15 \%$ Triton$\mathrm{X}$ in PBS, organoid sections were mounted with ProLong Diamond Antifade Mountant (Thermo Fisher Scientific) and sealed with nail polish. The slides were stored at $+4{ }^{\circ} \mathrm{C}$ until acquisition.

Confocal images of GFAP, TUJ1, and MAP2 staining were acquired on Fluoview FV10i (Olympus) confocal laser scanning microscope. Multiarea images were acquired with a 10x/NA 0.4 phase contrast objective in stack with z-step of $10 \mu \mathrm{m}$. Each acquisition allowed to collect the signals present in the entire 3D slice of the organoid. 
Images were analyzed on Fiji software quantifying the GFAP, MAP2, and TUJ1 signal as fluorescence intensity normalized for the area of the entire slice.

Confocal images of GFAP and MAP2 staining at higher magnification were acquired at the Olympus iX83 FluoView1200 laser scanning microscope. Stacks images were acquired with the UPLSAPO60XO NA1,35 oil objective (Olympus), zoom $2 \mathrm{x}$ and with z-step of $0,3 \mu \mathrm{m}$. Astrocytes density analysis was performed by manually counting the number of GFAP + Hoechst + cells, normalized on the acquired volume for each FOV. In order to check the astrocytes positivity the entire acquisition volume was analyzed at single cell level using Fiji software.

\section{Western blot}

Whole-cell protein extracts were prepared from samples lysed in RIPA buffer (150 mM NaCl, 5 mM EDTA, $50 \mathrm{mM}$ Tris Base, 1\% NP-40, 0.5\% Sodium Deoxycholate, 0.1\% Sodium Dodecyl Sulfate). Extracts were separated by electrophoresis on NuPAGE 4-12\% Bis-Tris gels (Thermo Fisher Scientific) in MOPS buffer (Thermo Fisher Scientific) and blotted onto nitrocellulose membrane (GE Healthcare). Immunoblots were incubated overnight with polyclonal rabbit anti-FMRP antibody (ab27455 Abcam, 1:1000) and for $1 \mathrm{~h}$ with monoclonal mouse anti-GAPDH antibody (sc-32233 Santa Cruz, 1:3000) as loading control. Membranes were then incubated with HRP-conjugated donkey anti-mouse IgG $(\mathrm{H}+\mathrm{L})$ (IS20404 Immunological Sciences, 1:5000) or donkey anti-rabbit $\operatorname{IgG}(\mathrm{H}+\mathrm{L})$ (IS20405 Immunological Sciences, 1:5000) secondary antibodies for $45 \mathrm{~min}$. HRP signal was revealed with the Clarity Western ECL Substrates kit (Bio-rad). Images were acquired with the Chemidoc MP (Bio-Rad) and analyzed with the ImageLab software (Bio-Rad).

\section{PCR, RT-PCR, and RT-qPCR}

Genomic DNA was extracted from cells using PCRBIO Rapid Extract PCR Kit (PCR Biosystems) according to manufacturer instructions. After extraction, genomic DNA was amplified for 40 cycles using oligos indicated in Supplementary Table 2.

Total RNA was extracted with the EZNA Total RNA Kit I (Omega Bio-Tek) and retrotranscribed using the iScript Reverse Transcription Supermix for RT-qPCR (Bio-Rad).

For FMRP-KO clones screening (Fig. 1C), target cDNA sequences were amplified for 35 reaction cycles with the enzyme MyTaq DNA Polymerase (Bioline). The cDNA of the housekeeping gene ATP5O (ATP synthase, $\mathrm{H}+$ transporting, mitochondrial F1 complex, $\mathrm{O}$ subunit), used as internal control, was amplified for 28 reaction cycles.

Real-time RT-PCR was performed with iTaq Universal SYBR Green Supermix (Bio-Rad) on a ViiA 7 Real-Time PCR System (Applied Biosystems). A complete list of primers is provided in Supplementary material (Supplementary Table 2).

\section{Calcium imaging recordings and data processing}

Fluorescence images were acquired at room temperature using a customized digital imaging microscope. Excitation of calcium dye was achieved at wavelenght $488 \mathrm{~nm}$ using the highly stable light source Lambda XL (Sutter Instrument) equipped with a Lambda 10-B optical filter changer (Sutter Instrument). For the collection of the emitted light, a 525/50 nm filter was used. Fluorescence was visualized using the Zeiss Axio observer A1 inverted microscope (Zeiss) equipped with a Zeiss A-Plan 10x/NA 0.25 infinity corrected objective (Zeiss) and a CoolSNAPHQ2 camera (Photometrics). Images acquisition at $4 \mathrm{~Hz}$ sampling rate were performed using Micromanager software. Changes in the intracellular $\mathrm{Ca}^{2+}$ level were observed using the high-affinity $\mathrm{Ca}^{2+}$-sensitive indicator Fluo4-AM (Invitrogen) which was used at the concentration of $5 \mu \mathrm{M}$ by incubating neuronal cultures for $30 \mathrm{~min}$ at $37^{\circ} \mathrm{C}$ in HEPES-buffered external solution (NES) containing $140 \mathrm{mM} \mathrm{NaCl}, 2.8 \mathrm{mM} \mathrm{KCl}, 2 \mathrm{mM}$ $\mathrm{CaCl}_{2}, 2 \mathrm{mM} \mathrm{MgCl}, 10 \mathrm{mM}$ HEPES, $10 \mathrm{mM}$ D-glucose (pH 7.3 with $\mathrm{NaOH} ; 290$ mOsm).

Calcium imaging data processing was performed through custom numeric codes implemented in MATLAB environment. Recorded images sequences were collected and saved as 3D matrix. The first step of analysis concerned the definition of regions of interest for neurons. We realized an automated algorithm to identify only active neurons by analyzing the cumulative difference of the signal between the various frames of the time series. The obtained matrix was studied in the frequency domain via $2 \mathrm{D}$ Fourier transform and suitably filtered to eliminate high-frequency noisy components; then the local maxima of the matrix were selected. The algorithm provided the possibility to manually adjust eventual false or missing detections. Once established active cell positions (no more than 200 cells selected for each field of view), their fluorescence signals as a function of time were collected. A supervised analysis allowed the detection of calcium transients and relative characteristics (amplitude, rising and decay time). In detail, the raw traces of the neurons extracted from the t-stack were baseline corrected and normalized as $\Delta F / F_{0}$; then smoothed using a moving average filter. On the filtered traces, a putative event was detected when a series of conditions were satisfied: at the onset the fluorescence intensity and the slope of the trace show an increase; at the offset the slope of the trace decreases and a certain time interval occurs within the onset and the offset ${ }^{61}$. Threshold for peaks amplitude was set to $1 \%$ of the baseline value. This operation gave a first indication about the onset time of each event, which was used as starting point for a fitting procedure based on a 
previously described algorithm ${ }^{62}$. The algorithm assumes that there exists an elementary calcium transient characteristic of a single action potential and that the calcium transients add up linearly. Each putative transient was fitted in two-step with a model function composed of a single-exponential rise and a single-exponential decay.

In the first step, we fitted the onset to determine the start of the event $t_{0}$ and the onset time constant $\tau_{\text {on }}$.

$$
I(t)=k\left(1-e^{-\frac{t-t_{0}}{\tau_{0}}}\right)
$$

Then we fitted the entire calcium transient to estimate amplitude and the decay component $\tau_{\text {off- }}$.

$$
I(t)=a \cdot e^{-\frac{t-t_{0}}{\tau_{\text {off }}}} \cdot\left(1-e^{-\frac{t-t_{0}}{\tau_{0 n}}}\right)
$$

The analysis of calcium dynamics was crucial to distinguish fast calcium transients, typical of neuronal activity, from calcium signals characterized by a slowest onset (release from internal stores, calcium signals from other cell types). According to our experimental conditions, we established a threshold $\tau^{*}=2 \mathrm{~s}$ to recognize and discard non-neuronal signals: neurons whose mean rising time results below this threshold were selected, all other signals were not considered for final statistics. Event rate, amplitude, and synchrony of the network (evaluated as the relative number of simultaneous events) were exported in Microsoft Excel.

For the agonist-evoked calcium activity, images were acquired at a frequency of $1 \mathrm{~Hz}$ for $15 \mathrm{~min}$ with an exposure time of $200 \mathrm{~ms}$ through a BX51WI microscope (Olympus Corporation, Tokyo, JP. Objectives: LUMPlanF $\mathrm{N} 10 \mathrm{x} / 0.10$, air, and 40x/0.80, water immersion, Olympus Corporation). Fluo4-AM was excited at $488 \mathrm{~nm}$ with an Optoscan monochromator (Cairn Research, Facersham, UK). Light was generated by a xenon lamp Optosource (Cairn Research). A borosilicated glass micropipette was filled with NES and $2 \mathrm{mM}$ Glutamate or $1 \mathrm{mM}$ GABA (Sigma Aldrich), and moved via a micromanipulator MP225 (Sutter Instruments, Novato, CA) to reach the core of the field of view, around $50 \mu \mathrm{m}$ beneath the surface of the $\operatorname{dish}^{63}$.

The basal fluorescence was evaluated for $5 \mathrm{~min}$, then a small volume of agonist-containing solution was puffed on the cells via a pneumatic pico-pump (PV820; World Precision Instruments, Inc., Sarasota, FL) with a brief pressure (10 psi; $100 \mathrm{~ms})$. The images were acquired with a camera CCD CoolSnap MYO (Photometrics, Tucson, AZ, USA) and then analyzed with MetaFluor software as fluorescence variation measured into regions of interest (ROI) corresponding to single cells. To quantify the signal, the formula $\left(F-F_{0}\right) / F_{0}$ was used, where $F_{0}$ is the average fluorescence before the agonist application and $F$ the fluorescence during the time-lapse acquisition.

\section{Statistical data analysis}

Statistical analysis, graphs, and plots were generated using GraphPad Prism 6 (GraphPad Software) and MATLAB 2016b (MathWorks). To verify whether our data sets were reflecting normal distribution, the Shapiro-Wilk normality test was performed. Where the normality distribution was not fulfilled, statistical significance analysis was performed using the nonparametric two-sided Mann-Whitney test (MW test, $P=0.05$ ). In all other cases, whether not stated otherwise, Student $t$ test $(P=0.05)$ was performed. Data set are given as mean \pm standard error of the mean (s.e.m.), the number of cells, replicats, fields of views, cultures, and organid batches are reported for each experiments in the cporresponding figure legend. Statistical significance of the cumulative distribution were calculated using the nonparametric Kolmogorov-Smirnov test.

\section{Acknowledgements}

We thank the members of Di Angelantonio, Rosa, and Reggiani laboratories for helpful discussion. The authors wish to thank the Imaging Facility at Center for Life Nano- \& Neuro- Science, Istituto Italiano di Tecnologia, for support and technical advice.

\section{Author details}

${ }^{1}$ Center for Life Nano- \& Neuro-Science, Istituto Italiano di Tecnologia, Viale Regina Elena 291, 00161 Rome, Italy. ${ }^{2}$ Department of Physiology and Pharmacology, Sapienza University of Rome,

P.le A. Moro 5, 00185 Rome, Italy. ${ }^{3}$ Department of Biology and Biotechnologies "Charles Darwin", Sapienza University of Rome, P.le A. Moro 5, 00185 Rome, Italy. ${ }^{4}$ Department of Physics, Sapienza University of Rome, P.le A. Moro 5, 00185 Rome, Italy. ${ }^{5}$ D3 Validation Research Line, Istituto Italiano di Tecnologia, Via Morego 30, 16163 Genova, Italy

\section{Author contributions}

Conceptualization, A.Ro., S.D.A., A.S., and A.Re.; Formal analysis, A.S., C.B., V.d.T., S.G., and M.R.; Investigation, C.B., F.S., A.S., F.C., V.d.T., and C.D.A.; Methodology, C.B., F.S., A.S., P.F.P., M.R., and F.C.; Project administration, A.Ro., S.D.A., and A.Re.; Supervision, S.D.A. and A.Ro.; Writing - original draft, S.D.A. and A.Ro.

\section{Funding}

This research was funded by the CrestOptics-IIT JointLab for Advanced Microscopy (to C.B, A.S. and S.D.A.) the MARBEL Life2020 Grant (to C.B. and S.D. A.), the Bio3DBrain FSE 2014-2020 Regione Lazio (to S.D.A.). This work was partially supported by Sapienza University and Fondazione Istituto Italiano di Tecnologia to A.Ro. and S.D.A.

\section{Code availability}

A custom code based on MathWorks Matlab 2015 software was used for analyzing calcium imaging data. The code is available upon request.

Ethics statement

Ethics approval was not required for this work.

\section{Conflict of interest}

The authors declare no conflict of interest. The funders had no role in the design of the study; in the collection, analyses, or interpretation of data; in the writing of the manuscript, or in the decision to publish the results. 


\section{Publisher's note}

Springer Nature remains neutral with regard to jurisdictional claims in published maps and institutional affiliations.

Supplementary information The online version contains supplementary material available at https://doi.org/10.1038/s41419-021-03776-8.

Received: 19 November 2020 Revised: 26 April 2021 Accepted: 28 April 2021

Published online: 15 May 2021

\section{References}

1. Martin, J. P. \& Bell, J. A pedigree of mental defect showing sex-linkage. J. Neurol. Neurosurg. Psychiatry 6, 154-157 (1943).

2. Hagerman, R. J. \& Hagerman, P. J. The fragile $X$ premutation: into the phenotypic fold. Curr. Opin. Genet. Dev. 12, 278-283 (2002).

3. Hunter, J. et al. Epidemiology of fragile $X$ syndrome: a systematic review and meta-analysis. Am. J. Med. Genet. Part A 164, 1648-1658 (2014).

4. Lozano, R., Rosero, C. A. \& Hagerman, R. J. Fragile X spectrum disorders. Intractable Rare Dis. Res. 3, 134-146 (2014)

5. Bagni, C., Tassone, F., Neri, G. \& Hagerman, R. Fragile $X$ syndrome: causes, diagnosis, mechanisms, and therapeutics. J. Clin. Invest. 122, 4314-4322 (2012).

6. Antar, L. N., Dictenberg, J. B., Plociniak, M., Afroz, R. \& Bassell, G. J. Localization of FMRP-associated mRNA granules and requirement of microtubules for activity-dependent trafficking in hippocampal neurons. Genes Brain Behav. 4, 350-359 (2005).

7. Richter, J. D., Bassell, G. J. \& Klann, E. Dysregulation and restoration of translational homeostasis in fragile X syndrome. Nat. Rev. Neurosci. 16, 595-605 (2015).

8. Contractor, A., Klyachko, V. A. \& Portera-Cailliau, C. Altered neuronal and circuit excitability in fragile X syndrome. Neuron 87, 699-715 (2015).

9. Banerjee, A., Ifrim, M. F., Valdez, A. N., Raj, N. \& Bassell, G. J. Aberrant RNA translation in fragile $X$ syndrome: from FMRP mechanisms to emerging therapeutic strategies. Brain Res. 1693, 24-36 (2018).

10. Till, S. M. et al. Altered maturation of the primary somatosensory cortex in a mouse model of fragile X syndrome. Hum. Mol. Genet. 21, 2143-2156 (2012).

11. Kooy, R. F. et al. Transgenic mouse model for the fragile $X$ syndrome. Am. J. Med. Genet. 64, 241-245 (1996)

12. $L i, Y . \&$ Zhao, $X$. Concise review: fragile $X$ proteins in stem cell maintenance and differentiation. Stem Cells 32, 1724-1733 (2014).

13. Liu-Yesucevitz, L. et al. Local RNA translation at the synapse and in disease. J. Neurosci. 31, 16086-16093 (2011).

14. Shi, Y., Inoue, H., Wu, J. C. \& Yamanaka, S. Induced pluripotent stem cell technology: a decade of progress. Nat. Rev. Drug Discov. 16, 115-130 (2017).

15. Doers, M. E. et al. IPSC-derived forebrain neurons from FXS individuals show defects in initial neurite outgrowth. Stem Cells Dev. 23, 1777-1787 (2014).

16. Halevy, T., Czech, C. \& Benvenisty, N. Molecular mechanisms regulating the defects in fragile $x$ syndrome neurons derived from human pluripotent stem cells. Stem Cell Rep. 4, 37-46 (2015).

17. Vershkov, D. et al. FMR1 reactivating treatments in fragile $X$ iPSC-derived neural progenitors in vitro and in vivo. Cell Rep. 26, 2531-2539 (2019). e4.

18. Sandoe, J. \& Eggan, K. Opportunities and challenges of pluripotent stem cell neurodegenerative disease models. Nat. Neurosci. 16, 780-789 (2013).

19. Paquet, D. et al. Efficient introduction of specific homozygous and heterozygous mutations using CRISPR/Cas9. Nature 533, 125-129 (2016).

20. Schöndorf D. C., et al. IPSC-derived neurons from GBA1-associated Parkinson's disease patients show autophagic defects and impaired calcium homeostasis. Nat. Commun. https://doi.org/10.1038/ncomms5028 (2014).

21. $\mathrm{Xu}, \mathrm{X}$. et al. Reversal of phenotypic abnormalities by CRISPR/Cas9-mediated gene correction in Huntington disease patient-derived induced pluripotent stem cells. Stem Cell Rep. 8, 619-633 (2017).

22. Hong, Y. J. \& Do, J. T. Neural lineage differentiation from pluripotent stem cells to mimic human brain tissues. Front. Bioeng. Biotechnol. 7, 1-17. (2019).

23. Thomas, C. H., Collier, J. H., Sfeir, C. S. \& Healy, K. E. Engineering gene expression and protein synthesis by modulation of nuclear shape. Proc. Natl Acad. Sci. USA 99, 1972-1977 (2002).

24. Vergani, L., Grattarola, M. \& Nicolini, C. Modifications of chromatin structure and gene expression following induced alterations of cellular shape. Int. J. Biochem. Cell Biol. 36, 1447-1461 (2004).
25. Wrzesinski, K. \& Fey, S. From 2D to 3D-a new dimension for modelling the effect of natural products on human tissue. Curr. Pharm. Des. 21, 5605-5616 (2015).

26. Knight, E. \& Przyborski, S. Advances in 3D cell culture technologies enabling tissue-like structures to be created in vitro. J. Anat. 227, 746-756 (2015).

27. Choi, S. H. et al. A three-dimensional human neural cell culture model of Alzheimer's disease. Nature 515, 274-278 (2014).

28. Simian, M. \& Bissell, M. J. Organoids: a historical perspective of thinking in three dimensions. J. Cell Biol. 216, 31-40 (2017).

29. Kelava, I. \& Lancaster, M. A. Dishing out mini-brains: current progress and future prospects in brain organoid research. Dev. Biol. 420, 199-209 (2016).

30. Mariani, J. et al. FOXG1-dependent dysregulation of GABA/glutamate neuron differentiation in autism spectrum disorders. Cell 162, 375-390 (2015).

31. Wang, P. et al. CRISPR/Cas9-mediated heterozygous knockout of the autism gene CHD8 and characterization of its transcriptional networks in cerebral organoids derived from iPS cells. Mol. Autism 8, 1-17. (2017).

32. Lenzi, J. et al. ALS mutant FUS proteins are recruited into stress granules in induced pluripotent stem cell-derived motoneurons. Dis. Model Mech. 8, 755-766 (2015)

33. Shi, Y., Kirwan, P. \& Livesey, F. J. Directed differentiation of human pluripotent stem cells to cerebral cortex neurons and neural networks. Nat. Protoc. 7 1836-1846 (2012)

34. Salaris, F. et al. 3D bioprinted human cortical neural constructs derived from induced pluripotent stem cells. J. Clin. Med. 8, 1595 (2019).

35. Ghirga, S. et al. Optonongenetic enhancement of activity in primary cortical neurons. J. Opt. Soc. Am. A 37, 643 (2020).

36. Sunamura, N., Iwashita, S., Enomoto, K., Kadoshima, T. \& Isono, F. Loss of the fragile $X$ mental retardation protein causes aberrant differentiation in human neural progenitor cells. Sci. Rep. 8, 1-13. (2018).

37. Lee, F. H. F., Lai, T. K. Y., Su, P. \& Liu, F. Altered cortical cytoarchitecture in the Fmr1 knockout mouse. Mol. Brain 12, 1-12. (2019).

38. Bülow, P., Murphy, T. J., Bassell, G. J. \& Wenner, P. Homeostatic intrinsic plasticity is functionally altered in Fmr1 KO cortical neurons. Cell Rep. 26, 1378-1388 (2019). e3.

39. Achuta, V. S., et al. Functional changes of AMPA responses in human induced pluripotent stem cell-derived neural progenitors in fragile $\mathrm{x}$ syndrome. Sci. Signal https://doi.org/10.1126/scisignal.aan8784 (2018).

40. Danesi, $C$. et al. Increased calcium influx through L-type calcium channels in human and mouse neural progenitors lacking fragile $X$ mental retardation protein. Stem Cell Rep. 11, 1449-1461 (2018).

41. Achuta, V. S. et al. Metabotropic glutamate receptor 5 responses dictate differentiation of neural progenitors to NMDA-responsive cells in fragile $X$ syndrome. Dev. Neurobiol. 77, 438-453 (2017).

42. Morin-Parent, F., Champigny, C., Lacroix, A., Corbin, F. \& Lepage, J.-F. Hyperexcitability and impaired intracortical inhibition in patients with fragile-X syndrome. Transl. Psychiatry 9, 312 (2019).

43. Braat, $S$. et al. The GABAA receptor is an FMRP target with therapeutic potential in fragile X syndrome. Cell Cycle 14, 2985-2995 (2015).

44. Sabanov, V. et al. Impaired GABAergic inhibition in the hippocampus of Fmr 1 knockout mice. Neuropharmacology 116, 71-81 (2017).

45. Kang, J. Y. et al. Deficits in the activity of presynaptic-aminobutyric acid type B receptors contribute to altered neuronal excitability in fragile $X$ syndrome. J. Biol. Chem. 292, 6621-6632 (2017).

46. Telias, M., Segal, M. \& Telias, M. Immature responses to GABA in fragile $X$ neurons derived from human embryonic stem cells. Front. Cell Neurosci. 10, 1-9 (2016).

47. Telias, M. Pharmacological treatments for fragile $X$ syndrome based on synaptic dysfunction. Curr. Pharm. Des. 25, 4394-4404 (2019).

48. Cellot, G. \& Cherubini, E. Functional role of ambient GABA in refining neuronal circuits early in postnatal development. Front. Neural Circuits 7, 1-9 (2013).

49. Palma, E. et al. Phosphatase inhibitors remove the run-down of $y$-aminobutyric acid type $A$ receptors in the human epileptic brain. Proc. Natl Acad. Sci. USA 101, 10183-10188 (2004).

50. Di Angelantonio, S. et al. A role for intracellular zinc in glioma alteration of neuronal chloride equilibrium. Cell Death Dis. 5, e1501-e1510 (2014).

51. He, Q. et al. Interneuron- and GABAA receptor-specific inhibitory synaptic plasticity in cerebellar Purkinje cells. Nat. Commun. 6, 1-13. (2015).

52. Wang $L$., et al. Imbalance between glutamate and GABA in Fmr1 knockout astrocytes influences neuronal development. Genes https://doi.org/10.3390/ genes7080045 (2016). 
53. Jacobs, S., Nathwani, M. \& Doering, L. C. Fragile $X$ astrocytes induce developmental delays in dendrite maturation and synaptic protein expression. BMC Neurosci. 11, 132 (2010).

54. Jacobs, S., Cheng, C. \& Doering, L. C. Hippocampal neuronal subtypes develop abnormal dendritic arbors in the presence of fragile $X$ astrocytes. Neuroscience 324, 202-217 (2016)

55. Cheng, C., Sourial, M. \& Doering, L. C. Astrocytes and developmental plasticity in fragile X. Neural Plast. https://doi.org/10.1155/2012/197491 (2012).

56. Guo, W. et al. Inhibition of GSK3 $\beta$ improves hippocampusdependent learning and rescues neurogenesis in a mouse model of fragile $X$ syndrome. Hum. Mol. Genet. 21, 681-691 (2012).

57. Jin, Y. H., Kim, H., Oh, M., Ki, H. \& Kim, K. Regulation of Notch1/NICD and Hes1 expressions by GSK-3a/ß. Mol. Cells 27, 15-19 (2009).

58. Morrison, S. J. et al. Transient notch activation initiates an irreversible switch from neurogenesis to gliogenesis by Neural Crest Stem Cells (NCSCs). Stem cells self-renew and can generate one or more classes of neurons, glia, and myofibroblasts (also known as smooth muscle cells). Cell 101, 499-510 (2000).

59. De Santis, R. et al. FUS mutant human motoneurons display altered transcriptome and microRNA pathways with implications for ALS pathogenesis. Stem Cell Rep. 9, 1450-1462 (2017).

60. Lancaster, M. A. \& Knoblich, J. A. Generation of cerebral organoids from human pluripotent stem cells. Nat. Protoc. 9, 2329-2340 (2014).

61. Palazzolo G., et al. Fast wide-volume functional imaging of engineered in vitro brain tissues. Sci Rep. https://doi.org/10.1038/s41598-017-08979-8 (2017).

62. Grewe, B. F., Langer, D., Kasper, H., Kampa, B. M. \& Helmchen, F. High-speed in vivo calcium imaging reveals neuronal network activity with nearmillisecond precision. Nat. Methods 7, 399-405 (2010).

63. Di Angelantonio, S. \& Nistri, A. Calibration of agonist concentrations applied by pressure pulses or via rapid solution exchanger. J. Neurosci. Methods 110, 155-161 (2001). 